\title{
Simultaneous search for extra light and heavy Higgs bosons via cascade decays
}

\section{Ulrich Ellwanger and Matías Rodríguez-Vázquez}

Laboratoire de Physique Théorique, UMR 8627, CNRS, Université de Paris-Sud, Université Paris-Saclay, 91405 Orsay, France

E-mail: ellwanger@th.u-psud.fr, matias.rodriguez-vazquez@th.u-psud.fr

ABSTRACT: Models with extended Higgs sectors can contain several additional Higgs states, heavier or lighter than the SM Higgs boson. The couplings of lighter extra states to SM particles can be strongly reduced, leading to small cross sections for their direct production. Heavier extra states can have larger couplings to SM particles and, moreover, have large branching fractions into lighter extra states, notably into a SM-like Higgs boson accompagnied by another Higgs state which can be lighter or heavier than $125 \mathrm{GeV}$. Motivated by corresponding scenarios in the NMSSM we study the prospects for the discovery or exclusion of cascade decays $g g F \rightarrow H_{3} \rightarrow H_{2}+H_{1}$ in the $b \bar{b} b \bar{b}, b \bar{b} \tau \tau$ and $b \bar{b} \gamma \gamma$ final states where either $H_{1}$ or $H_{2}$ can be SM-like. Significant regions of the NMSSM parameter space can be tested by these searches. These are, however, not confined to models of the NMSSM type.

KEywords: Higgs Physics, Beyond Standard Model, Supersymmetric Standard Model

ARXIV EPRINT: 1707.08522 


\section{Contents}

1 Introduction $\quad 1$

2 The neutral Higgs sector of the NMSSM 3

3 Simulation of signal samples 5

4 Search strategy for the $b \bar{b} b \bar{b}$ final state $\quad 6$

4.1 Analyses of signal samples $\quad 6$

$\begin{array}{llr}4.2 & \text { Background estimation } & 9\end{array}$

4.3 Future $95 \%$ CL exclusion limits and $5 \sigma$ discovery cross sections 11

5 Search strategies for the $b \bar{b} \tau \tau$ final state $\quad 13$

$\begin{array}{ll}5.1 \text { Analyses of signal samples } & 14\end{array}$

$\begin{array}{lll}5.2 & \text { Background estimation } & 17\end{array}$

5.3 Future 95\% CL exclusion limits and $5 \sigma$ discovery cross sections 18

6 Search strategies for the $b \bar{b} \gamma \gamma$ final state $\quad 21$

6.1 Analyses of signal samples 21

6.2 Background estimation 22

6.3 Future 95\% CL exclusion limits and $5 \sigma$ discovery cross sections 23

$\begin{array}{llr}7 & \text { Conclusions and outlook } & 26\end{array}$

$\begin{array}{ll}\text { A Functions to fit background distributions } & 28\end{array}$

B $5 \sigma$ discovery and $95 \%$ CL exclusion limits 29

\section{Introduction}

Extended Higgs sectors are frequent properties of models beyond the Standard Model (BSM). Such extra states can have very small couplings to quarks, leptons and SM gauge fields. For instance, for singlets under the SM gauge symmetries such renormalizable couplings are disallowed by gauge invariance. The direct production cross sections for these states are then strongly suppressed in all channels. On the other hand, couplings of singlets to $\mathrm{SU}(2)$ Higgs doublets of the SM- or BSM-type are possible and typically present in BSM models. This allows for the discovery of such states in cascade decays of heavy BSM SU(2) Higgs doublets, provided the production cross sections of the latter are larg enough. 
The final states after BSM-Higgs to BSM-Higgs + SM-Higgs cascades typically correspond to the ones in searches for resonant SM-Higgs $\left(H_{125}\right)$ pair production: mainly $b \bar{b} b \bar{b}$, $b \bar{b} \tau \tau$ and $b \bar{b} \gamma \gamma$. Corresponding searches have been performed at the LHC by ATLAS [1-6] and by CMS [7-21]. However, one of the SM-like Higgs bosons would now be replaced by a lighter or heavier BSM-Higgs boson. One can argue that the cross sections for such processes can be more promising than for resonant SM-Higgs pair production:

a) A sizeable gluon-gluon-fusion $(g g F)$ production cross section of a heavy scalar or pseudoscalar $\Phi$, i.e. a sizeable coupling of $\Phi$ to top quarks, requires $\Phi$ to possess a sizeable $\mathrm{SU}(2)$-doublet component. However, since $H_{125}$ is also a $\mathrm{SU}(2)$-doublet, trilinear couplings $\Phi-H_{125}-H_{125}$ (with $\Phi$ a pure doublet) violate the $\mathrm{SU}(2)$ symmetry and must be proportional to a $\mathrm{SU}(2)$ symmetry breaking vev; the latter is limited from above by the $Z / W$ masses. This limits the possible partial width for $\Phi \rightarrow H_{125}+H_{125}$, whereas the concurrent decay $\Phi \rightarrow t \bar{t}$ is always possible if $\Phi$ can be produced in $g g F$ and is heavier than $2 m_{\text {top }}$.

b) In the case $g g F \rightarrow \Phi \rightarrow H_{125}+H^{\prime}$ with $\Phi$ a pure doublet, the trilinear coupling $\Phi-H_{125}-H^{\prime}$ can be $\mathrm{SU}(2)$ invariant if $H^{\prime}$ is a singlet. In models with extended Higgs sectors including both an extra doublet and a singlet, such a coupling can thus be much larger than the $Z / W$ masses leading to sizeable $\Phi \rightarrow H_{125}+H^{\prime}$ partial widths.

In Two-Higgs-Doublet-Models of type II such as the Minimal Supersymmetric Standard Model (MSSM) the production cross sections for extra CP-even $(H)$ and CP-odd $(A)$ Higgs doublets are not suppressed, and are dominated by $g g F$ for $\tan \beta$ not too large [2224]. $H$ or $A$ can thus play the rôle of $\Phi$ above. The Next-to-Minimal Supersymmetric Standard Model (NMSSM) $[25,26]$ contains additional CP-even $\left(H_{S}\right)$ and CP-odd $\left(A_{S}\right)$ singletlike states with masses typically below $M_{H} \sim M_{A}$. One finds that the $B R\left(H \rightarrow H_{S}+H_{125}\right)$ and $B R\left(A \rightarrow A_{S}+H_{125}\right)$ can be up to $\sim 50 \%$ [27-31], for the reasons given above and detailed in the next section.

In the NMSSM this offers the possibility to produce otherwise practically invisible mostly singlet-like states $H_{S} / A_{S}$ in cascade decays of $H / A$ [27-31]. It is the aim of the present paper to study the prospects for discovery or exclusion of, simultaneously, $H / A$ and $H_{S} / A_{S}$ states in $g g F \rightarrow H \rightarrow H_{S}+H_{125}$ or $g g F \rightarrow A \rightarrow A_{S}+H_{125}$ in the final states $b \bar{b} b \bar{b}, b \bar{b} \tau \tau$ and $b \bar{b} \gamma \gamma$. Supersymmetry plays no rôle here, accordingly our results are applicable to any models with similarly extended Higgs sectors; see, e.g., [32].

We will adopt various strategies from the searches for resonant SM Higgs pair production by ATLAS [1-6] and by CMS [7-21]. Moreover, for $M_{H_{S}}$ near $125 \mathrm{GeV}$ we can compare our backgrounds and expected 95\% CL upper limits on the cross sections times branching fractions to the ones obtained in these publications.

On the other hand, the analyses presented here are complicated by the fact that the masses $M_{H_{S}} / M_{A_{S}}$ are not known a priori. An important aspect of optimal search strategies are $M_{H_{S}} / M_{A_{S}}$ dependent selection criteria (cuts) on events, hence different analyses should be performed, varying the assumptions on $M_{H_{S}} / M_{A_{S}}$. Only at the end of each of these 
analyses a search for a resonance-like bump in the total invariant mass of the $H_{S} / A_{S}$ plus $H_{125}$ decay products, which should correspond to $M_{H} / M_{A}$, is proposed.

In the next section we discuss shortly the Higgs sector of the NMSSM and the couplings relevant for the processes considered here. In section 3 we present features of our signal simulations. In section 4 we discuss the optimal search strategy for the $b \bar{b} b \bar{b}$ final state, and compare expected 95\% CL upper limits and $5 \sigma$ discovery limits on the cross sections times branching fractions to the ones possible in the NMSSM. Sections 5 and 6 are devoted to the $b \bar{b} \tau \tau$ and $b \bar{b} \gamma \gamma$ final states. All these search strategies and results are identical for $g g F \rightarrow H \rightarrow H_{S}+H_{125}$ and $g g F \rightarrow A \rightarrow A_{S}+H_{125}$, for notational simplicity we will refer to $H \rightarrow H_{S}+H_{125}$ only. In section 7 we conclude with a summary and an outlook.

\section{The neutral Higgs sector of the NMSSM}

In this section we discuss briefly some properties of the Higgs sector of the CP-conserving $\mathbb{Z}_{3}$-invariant NMSSM. It consists in two $\mathrm{SU}(2)$ doublets $H_{u}, H_{d}$ and a complex singlet $S$. The superpotential of the Higgs sector reads

$$
W_{\text {Higgs }}=\lambda \hat{S} \hat{H}_{u} \cdot \hat{H}_{d}+\frac{\kappa^{3}}{3} \hat{S}^{3}
$$

where $\lambda$ and $\kappa$ are dimensionless Yukawa couplings, and $\hat{H}_{u}, \hat{H}_{d}$ and $\hat{S}$ denote chiral superfields. Once the real component of the superfield $\hat{S}$ develops a vacuum expectation value (vev) $s$, the first term in the superpotential generates an effective $\mu$ term

$$
\mu=\lambda s .
$$

The vev $v_{u}$ of $H_{u}$ generates up-type quark masses, the vev $v_{d}$ of $H_{d}$ generates masses for down-type quarks and leptons, and both vevs contribute to the $Z$ and $W^{ \pm}$masses. Their ratio is $\tan \beta=v_{u} / v_{d}$.

Decays of a heavy Higgs state into two lighter Higgs states occur in the presence of corresponding trilinear Higgs couplings. Most of the trilinear Higgs couplings in the $\mathbb{Z}_{3}$-invariant NMSSM originate from quartic terms in the Higgs potential (see [25, 26]) proportional to two powers of $\lambda, \kappa$ or the electroweak gauge couplings, once the (neutral) Higgs fields are expanded around their vevs and decomposed into their real and imaginary parts:

$$
H_{u}^{0}=v_{u}+\frac{1}{\sqrt{2}}\left(H_{u, r}^{0}+i H_{u, i}^{0}\right), \quad H_{d}^{0}=v_{d}+\frac{1}{\sqrt{2}}\left(H_{d, r}^{0}+i H_{d, i}^{0}\right), \quad S=s+\frac{1}{\sqrt{2}}\left(S_{r}+i S_{i}\right) .
$$

Hence the trilinear couplings are proportional to the vevs $v_{u}, v_{d}$ or $s$. Whereas $v_{u}, v_{d}$ are limited from above by $M_{Z}^{2}=\frac{g_{1}^{2}+g_{2}^{2}}{2}\left(v_{u}^{2}+v_{d}^{2}\right)$, a large vev $s$ can generate a trilinear coupling $\sim H_{u} \cdot H_{d} S$. Another source for such a coupling is a trilinear Higgs-dependent soft SUSY breaking term

$$
\lambda A_{\lambda} H_{u} \cdot H_{d} S+\text { h.c. }
$$

where the dimensionful parameter $A_{\lambda}$ can be much larger than Higgs vevs.

In order to obtain its impact on trilinear couplings among Higgs mass eigenstates, the mass matrices have to be diagonalized. In the CP-even sector, where one deals with a $3 \times 3$ 
mass matrix, a first step in this direction is a rotation in the $\mathrm{SU}(2)$ doublet sector into the so-called Higgs basis

$$
H_{u, r}^{0}=\sin \beta h^{\prime}-\cos \beta H^{\prime}, \quad H_{d, r}^{0}=\cos \beta h^{\prime}+\sin \beta H^{\prime}
$$

where the vev of $H^{\prime}$ is zero, and the vev $\left\langle h^{\prime}\right\rangle=\sqrt{v_{u}^{2}+v_{d}^{2}}$ is equal to the one of the SM Higgs boson. In fact, in most of the phenomenological acceptable regions of the parameter space of the NMSSM (near the alignment limit [29]) the eigenstates of the full $3 \times 3$ CP-even mass matrix are not very different from $h^{\prime}, H^{\prime}$ and $S_{r}$, and will be denoted by $H_{125}\left(\sim h^{\prime}\right.$, approximately SM-like), $H\left(\sim H^{\prime}\right.$, approximately MSSM-like) and $H_{S}\left(\sim S_{r}\right.$, approximately singlet-like) in the following.

The corresponding rotation of the imaginary components $H_{u, i}^{0}$ and $H_{d, i}^{0}$ (with $\beta \rightarrow-\beta$ ) diagonalizes their $2 \times 2$ mass submatrix exactly and generates the Goldstone boson together with the MSSM-like pseudoscalar $A^{\prime}$. The latter still mixes with the singlet-like $S_{i}$, but typically both differ little from the mass eigenstates $A$ and $A_{S}$.

Performing the rotation (2.5) in (2.4) and using the previous approximations in the $\mathrm{CP}$-even and $\mathrm{CP}$-odd sectors, one obtains the trilinear couplings

$$
\frac{\lambda A_{\lambda}}{\sqrt{2}}\left(\frac{\tan ^{2} \beta-1}{\tan ^{2} \beta+1} H_{125}\left(H H_{S}-A A_{S}\right)+\ldots\right)
$$

where the omitted terms are suppressed by $\tan \beta$. Hence, for not too small $\tan \beta \rightarrow$ 1, trilinear couplings $g_{H_{125} H H_{S}}$ and $g_{H_{125} A A_{S}}$ are generated which have no analog in the MSSM, and are larger than all other trilinear Higgs couplings if $\lambda A_{\lambda}$ is large.

On the other hand the masses $M_{H / A}$ of the nearly degenerate mostly MSSM-like states $H / A$ are approximatively given by

$$
M_{H / A}^{2} \sim \mu\left(A_{\lambda}+\frac{\kappa}{\lambda} \mu\right) \frac{1+\tan ^{2} \beta}{\tan \beta},
$$

which limits $A_{\lambda}$ from above for fixed $M_{H / A}, \tan \beta$, small $|\kappa / \lambda|$ and $|\mu| \gtrsim 100 \mathrm{GeV}$ (as required by the lower LEP bound on higgsino-like charginos). Accordingly the trilinear couplings $g_{H_{125} H H_{S}}$ and $g_{H_{125} A A_{S}}$ can be larger for larger $M_{H / A}^{2}$.

The production cross sections for the mostly MSSM-like states $H / A$ is dominated by $g g F[22-24]$; at $\sqrt{s}=13-14 \mathrm{TeV}$ and for $\tan \beta \sim 2-3$ (typical in the NMSSM) they are $\gtrsim \mathcal{O}(1 \mathrm{pb})$ up to $M_{H / A} \sim 600 \mathrm{GeV}$. The trilinear couplings $g_{H_{125} H H_{S}}$ and $g_{H_{125} A A_{S}}$ induce the decays $H \rightarrow H_{125}+H_{S}$ and $A \rightarrow H_{125}+A_{S}$ if kinematically allowed. The branching fractions $B R\left(H / A \rightarrow H_{125}+H_{S} / A_{S}\right)$ can be as large as $\sim 50 \%$, in contrast to the decay $H \rightarrow H_{125}+H_{125}$.

The singlet-like states $\Phi_{S}=H_{S} / A_{S}$ have small couplings to quarks, leptons and gauge fields induced by mixings with $h^{\prime}, H^{\prime}$ and $A^{\prime}$. Hence the production cross sections for $\Phi_{S}$ are typically small, and their discovery may have to rely on $H / A \rightarrow H_{125}+\Phi_{S}$ decays. Via the couplings induced by mixing, $\Phi_{S}$ can decay into the same channels as $H_{125}$ and $H / A$. For $M_{\Phi_{S}}>2 m_{\text {top }}$, decays into $t \bar{t}$ are dominant, whereas decays $\Phi_{S} \rightarrow b \bar{b}$ dominate typically for $M_{\Phi_{S}}<2 m_{\text {top }}$. (The branching ratio for $H_{S} \rightarrow W^{+} W^{-}$can also be sizeable [29].) For 
$M_{H_{S}}>250 \mathrm{GeV}$, decays $H_{S} \rightarrow H_{125}+H_{125}$ are possible, leading to double-resonant triHiggs production (not considered here). Decays $\Phi_{S} \rightarrow \tau^{+}+\tau^{-}$are practically always possible. In the regions in the NMSSM parameter space with all present constraints on the signal rates of $H_{125}$ being satisfied the $B R\left(\Phi_{S} \rightarrow \gamma+\gamma\right)$ is in the $0.1-0.3 \%$ range, making this decay observable as well. Henceforth we will consider resonant $b \bar{b} b \bar{b}, b \bar{b} \tau^{+} \tau^{-}$and $b \bar{b} \gamma \gamma$ final states originating from $\Phi_{S} \rightarrow b \bar{b}, \Phi_{S} \rightarrow \tau^{+} \tau^{-}$and $\Phi_{S} \rightarrow \gamma \gamma$ decays.

Of interest will be the product of cross sections times branching fractions $\sigma(g g F \rightarrow$ $H / A) \times B R\left(H / A \rightarrow H_{125}+H_{S} / A_{S} \rightarrow b \bar{b} b \bar{b}, b \bar{b} \tau^{+} \tau^{-}\right.$and $\left.b \bar{b} \gamma \gamma\right)$ for various masses $M_{H}$, $M_{H_{S}}$ and $M_{A_{S}}$, for realistic regions in the parameter space of the NMSSM. To this end we have performed scans using the public code NMSSMTools_5.1.0 [33,34] including the radiative corrections from [35]. All phenomenological constraints, including the absence of Landau singularities below the GUT scale and, notably, constraints from Higgs searches in various channels at LEP and LHC are applied.

These include searches for scalar and pseudoscalar Higgs production at LEP (including unconventional Higgs decays), constraints from $B$-physics, constraints on the mass of $H_{125}$ ( $\pm 3 \mathrm{GeV}$ to account for theoretical uncertainties) and on its signal rates from the combined ATLAS and CMS run I results which disallow too large $H_{125}-H_{S}$ mixings, constraints from searches for $g g F \rightarrow H_{S} \rightarrow \gamma \gamma$ for $M_{H_{S}}=65-122 \mathrm{GeV}$, and searches for $H / A$ in the $H / A \rightarrow \tau \tau$ channel with $H / A$ produced in association with $b$-quarks and via $g g F$. We note that constraints from these latter searches in the $M_{A}-\tan \beta$ plane are weak for $\tan \beta \approx 2-3$, typical in the NMSSM, and further alleviated if $H / A$ have large branching fractions into the final states considered here.

The results of these scans for $\sigma(g g F \rightarrow H / A) \times B R\left(H / A \rightarrow H_{125}+H_{S} / A_{S} \rightarrow\right.$ $b \bar{b} b \bar{b}, b \bar{b} \tau^{+} \tau^{-}$and $\left.b \bar{b} \gamma \gamma\right)$ will be compared to the sensitivities in different final states in the following sections. The $g g F$ production cross sections for $H / A$ have been obtained from the CERN Yellow Report web page [36] at NNLO+NNLL, after an appropriate rescaling of the $H / A$-gluon-gluon coupling provided by NMSSMTools_5.1.0. Also the branching ratios of $H / A$ and $H_{S} / A_{S}$ are taken from NMSSMTools_5.1.0. In the figures showing the 95\% CL exclusion limits and $5 \sigma$ discovery cross sections, viable values for the cross sections times branching fractions in the parameter space of the NMSSM will be indicated as light shaded blue regions for $g g F \rightarrow H \rightarrow H_{125}+H_{S}$, and as light shaded red regions for $g g F \rightarrow A \rightarrow H_{125}+A_{S}$. For simplicity we will use the notation $g g F \rightarrow H \rightarrow H_{125}+H_{S}$ for the search strategies; the same search strategies apply to $g g F \rightarrow A \rightarrow H_{125}+A_{S}$.

\section{Simulation of signal samples}

Signal events for the production of $H$ in $g g F$ are generated by MadGraph5_aMC@NLO [37] with matrix elements at NLO taken from aMC_SusHi [38-40] using the NNPDF2.3NLO PDF set [41] in the 4-flavour scheme. Renormalization and factorization scales are chosen as $H_{T} / 2$ on an event-wise basis. Pythia6.4 [42] is used for the $H \rightarrow H_{125}+H_{S}$ decays, the $H_{125}$ and $H_{S}$ decays and the subsequent showering and hadronization. The total widths of $H$ are below $M_{H} / 50$ in all cases, below $M_{H} / 100$ for $M_{H}<500 \mathrm{GeV}$, hence the narrow width approximation is well satisfied. 
Separate signal events have been generated for each pair $\left(M_{H}, M_{H_{S}}\right)$. For $M_{H}$ we chose $M_{H}=425,500,625,750$ and $1000 \mathrm{GeV}$. We varied $M_{H_{S}}$ in steps of $10 \mathrm{GeV}$ in the range $25-225 \mathrm{GeV}$, and in steps of $20 \mathrm{GeV}$ above $225 \mathrm{GeV}$ up to the kinematic boundary $M_{H}-125 \mathrm{GeV}$ (except for $M_{H}=1000 \mathrm{GeV}$ where $M_{H_{S}}$ was varied in steps of $25 / 50 \mathrm{GeV}$ ).

For each pair $\left(M_{H}, M_{H_{S}}\right)$ we generated $150 \mathrm{k}$ unweighted events, more than the expected number of events at $3000 \mathrm{fb}^{-1}$. Accordingly the statistical fluctuations from the Monte Carlo (MC) generation are small compared to the expected statistical fluctuations from the data; the latter will be taken into account.

The output is given to the detector simulation Delphes 3 [43]. Jets are clustered with Fast Jet 3.0.1 [44] using the anti- $k_{T}$ algorithm with $\Delta R=0.4$. For $b$-tagging the ATLAS card is used in Delphes 3.

The $p_{T}$ dependence of the $b$-tagging and mistagging efficiencies is chosen in the ATLAS card following the parametrizations given in [45]. The default value of the parameters in Delphes 3.3.2 correspond to a working point $\varepsilon_{b}=70 \%$ as, e.g., in the ATLAS search for Higgs pair production in the $4 b$ final state at $13 \mathrm{TeV}$ in [6]. We will employ the same settings except for the $b \bar{b} \gamma \gamma$ final state.

\section{Search strategy for the $b \bar{b} b \bar{b}$ final state}

Searches for resonant SM Higgs pair production in the $b \bar{b} b \bar{b}$ final state have been performed before by ATLAS at $8 \mathrm{TeV}[1,3,4]$ and at $13 \mathrm{TeV}[6]$, and by CMS at $8 \mathrm{TeV}[8,10,12]$ and at $13 \mathrm{TeV}[14,15]$.

Searches for $g g F \rightarrow H \rightarrow H_{S}+H_{125} \rightarrow b \bar{b} b \bar{b}$ are complicated by the presence of two unknown masses of $H$ and $H_{S}$. A naive approach would be to require one $b \bar{b}$ pair with a mass near $125 \mathrm{GeV}$, and to look for simultaneous excesses in the plane of invariant masses of the other $b \bar{b}$ pair and the total $4 b$ invariant mass. However, this approach does not allow to optimize cuts as function of different masses of $H$ and $H_{S}$. An at least $\sim 20 \%$ gain in efficiency can be obtained as follows:

a) Choose a tentative value for $M_{H_{S}}$, and optimise the cuts and the choice of $b \bar{b}$ pairs as function of this value;

b) Search subsequently for an excess in the total $4 b$ invariant mass (suitably corrected, see below).

Subsequently we describe first our simulation of signal samples and the strategies for the analysis. In the following subsections we discuss the background simulation and validation, and finally the results for the expected 95\% CL upper limits and $5 \sigma$ discovery limits on the cross sections times branching fractions as function of $M_{H}$ and $M_{H_{S}}$. The latter are compared to possible production cross sections times branching fractions in the NMSSM.

\subsection{Analyses of signal samples}

After the simulation of signal samples as described in section 3, at least four $b$-tagged jets with $p_{T}>30 \mathrm{GeV}$ and $|\eta|<2.5$ are required. Four $b$-tagged jets can be paired in 

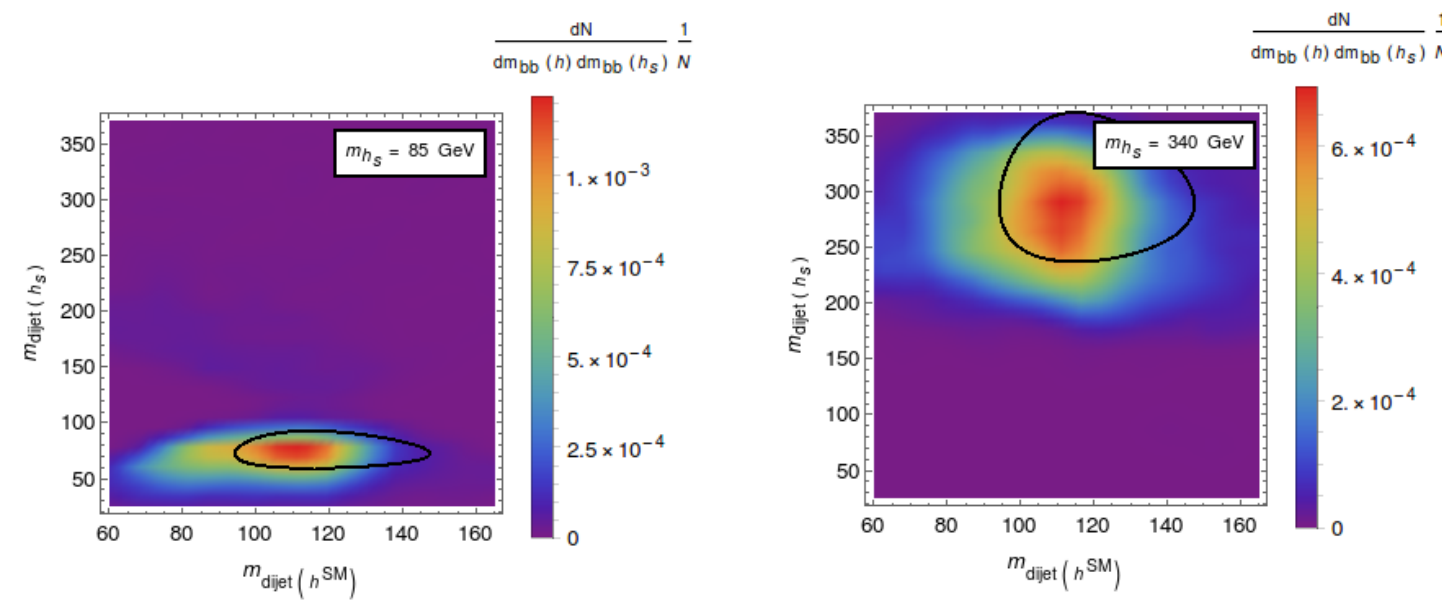

Figure 1. Dijet mass distributions $M_{b \bar{b}}\left(H_{125}\right)$ and $M_{b \bar{b}}\left(H_{S}\right)$ for two different benchmark points $M_{H_{S}}=85 \mathrm{GeV}$ and $M_{H_{S}}=340 \mathrm{GeV}$. The black contours indicate the signal regions defined by $\chi<2$.

six different ways. The two invariant masses of two $b \bar{b}$ pairs are tentatively denoted by $M_{b \bar{b}}\left(H_{125}\right)$ and $M_{b \bar{b}}\left(H_{S}\right)$. The subsequent procedure depends on the chosen value for $M_{H_{S}}$, and has to be repeated for each choice.

An event is kept only if a pairing exists for which $M_{b \bar{b}}\left(H_{125}\right)$ is sufficiently close to $125 \mathrm{GeV}$, and $M_{b \bar{b}}\left(H_{S}\right)$ is sufficiently close to $M_{H_{S}}$. In practice, the measured invariant masses of $b \bar{b}$ pairs are often somewhat smaller than the mass of the decaying Higgs boson. Therefore "sufficiently close to" should better be replaced by "slightly below" as in $[6,14]$. Generalizing the conditions applied in $[6,14]$, an event is kept only if a pairing exists for which

$$
\chi=\sqrt{\left(\frac{M_{b \bar{b}}\left(H_{125}\right)-115 \mathrm{GeV}}{0.1 M_{b \bar{b}}\left(H_{125}\right)}\right)^{2}+\left(\frac{M_{b \bar{b}}\left(H_{S}\right)-0.85 M_{H_{S}}}{0.1 M_{b \bar{b}}\left(H_{S}\right)}\right)^{2}}<2 .
$$

If different pairings within a given event satisfy (4.1), the combination that minimizes $\chi$ is chosen. In figures 1 we show, for $M_{H}=500 \mathrm{GeV}$, the distributions of the dijet masses $M_{b \bar{b}}\left(H_{125}\right)$ and $M_{b \bar{b}}\left(H_{S}\right)$ for the pairing minimizing $\chi$, for two different benchmark points $M_{H_{S}}=85 \mathrm{GeV}$ and $M_{H_{S}}=340 \mathrm{GeV}$ in the case where $M_{H_{S}}$ for the analysis was chosen correctly. The black contours indicate the signal regions defined by $\chi<2{ }^{1}$

In the case of heavy resonances with masses above $\sim 1 \mathrm{TeV}$, the two b-jets from a single Higgs boson tend to merge into a single fat jet. Accordingly a "boosted" analysis based on single $\Delta R=1.0$ jets per Higgs boson was applied in [6] for searches for such heavy resonances. We found that for such heavy $H$ states the production cross sections become too small for reasonable sensitivities, and limit ourselves to $M_{H} \leq 1 \mathrm{TeV}$ subsequently.

\footnotetext{
${ }^{1}$ An alternative pairing algorithm based on the angular distances of the constituent $b$-jets was studied. We found that the resulting sensitivities are mostly similar to those obtained using the mass-based algorithm described above when the jets are sufficiently collimated. However, as $M_{H_{S}}$ increases, the jets become too back-to-back, making the angular-pairing contraproductive. For this reason, we used the mass-based algorithm for the reconstruction of the Higgs candidates.
} 

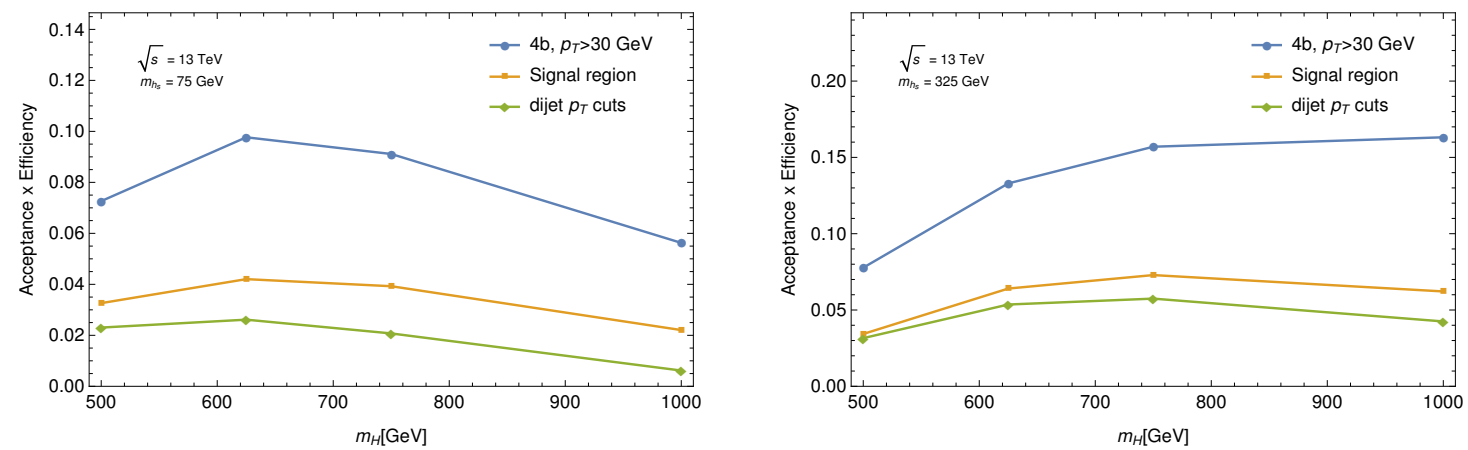

Figure 2. Efficiencies of the three cuts ( $4 b$ with $p_{T}>30 \mathrm{GeV}$ ), signal region (4.1) and dijet $p_{T}(4.2)$ for $M_{H_{S}}=75 \mathrm{GeV}$ and $M_{H_{S}}=325 \mathrm{GeV}$ as function of $M_{H}$.

We had tried to vary the jet reconstruction parameter $\Delta R$ without observing, however, a major impact on the possible sensitivities. The next step in the event selection are cuts on $p_{T}$ of the $b \bar{b}$ pairs associated with $H_{125}$ and $H_{S}$, respectively.

These cuts can be optimised with respect to $M_{4 b}$ (defined by the event) and the tentative value for $M_{H_{S}}$. To this end we considered many samples of $M_{H} \sim M_{4 b}$ and $M_{H_{S}}$. In each case we studied the dependence of the sensitivity $\epsilon_{s} / \sqrt{\epsilon_{B}}\left(\epsilon_{s}\right.$ and $\epsilon_{B}$ denote the efficiencies of the signal and background, respectively) on the cuts on $p_{T}$. Maximizing the sensitivities, we obtain different values for the optimal cuts on $p_{T}$ for each sample of $M_{4 b}$ and $M_{H_{S}}$. These different values for the cuts on $p_{T}$ are well approximated by the following functions of $M_{4 b}$ and $M_{H_{S}}$ :

$$
\begin{gathered}
p_{T}\left(b \bar{b}\left(H_{125}\right)\right)>1.6 \mathrm{GeV}+0.4 M_{4 b}-0.13 M_{H_{S}}-\frac{M_{H_{S}}}{M_{4 b}} \cdot 160 \mathrm{GeV} \\
p_{T}\left(b \bar{b}\left(H_{S}\right)\right)>12 \mathrm{GeV}+0.4 M_{4 b}-0.15 M_{H_{S}}-\frac{M_{H_{S}}}{M_{4 b}} \cdot 166 \mathrm{GeV}
\end{gathered}
$$

The efficiencies of the three cuts a) $4 b$ with $p_{T}>30 \mathrm{GeV}$, b) signal region (4.1) and c) dijet $p_{T}$ (4.2) are shown in figures 2 for $M_{H_{S}}=75 \mathrm{GeV}$ and $M_{H_{S}}=325 \mathrm{GeV}$ as function of $M_{H}$, and in figures 3 for $M_{H}=625 \mathrm{GeV}$ and $M_{H}=1000 \mathrm{GeV}$ as function of $M_{H_{S}}$. One observes a decrease of the efficiency of the condition of four $b$-tagged jets for $M_{H_{S}} \lesssim 80 \mathrm{GeV}$ (for $M_{H}=625 \mathrm{GeV}$ ) and $M_{H_{S}} \lesssim 100 \mathrm{GeV}$ (for $M_{H}=1000 \mathrm{GeV}$ ). Here the two $b$-jets from $H_{S}$ are boosted and hardly get resolved by the standard jet clustering algorithm; dedicated boosted analyses as in [6] could be envoked for this configuration.

For the search for a resonance $H$ we found it useful to replace $M_{4 b}$ by $M_{X}$, with $M_{X}$ defined such that uncertainties in the measurements of $M_{b \bar{b}}$ relative to $M_{H_{125}}$ and $M_{H_{S}}$ (typically due to radiation out of the jet cones) are corrected:

$$
M_{X}=M_{4 b}+125 \mathrm{GeV}-M_{b \bar{b}}\left(H_{125}\right)+M_{H_{S}}-M_{b \bar{b}}\left(H_{S}\right) .
$$

The empirical variable $M_{X}$ was already used by CMS in resonant double Higgs production search in the $b \bar{b} \gamma \gamma$ channel [14]. We found that replacing $M_{X}$ by a full Lorentz covariant expression in terms of $M_{H_{125}}, M_{H_{S}}$ and the two values of $M_{b \bar{b}}$ did not improve the sensitivities. 

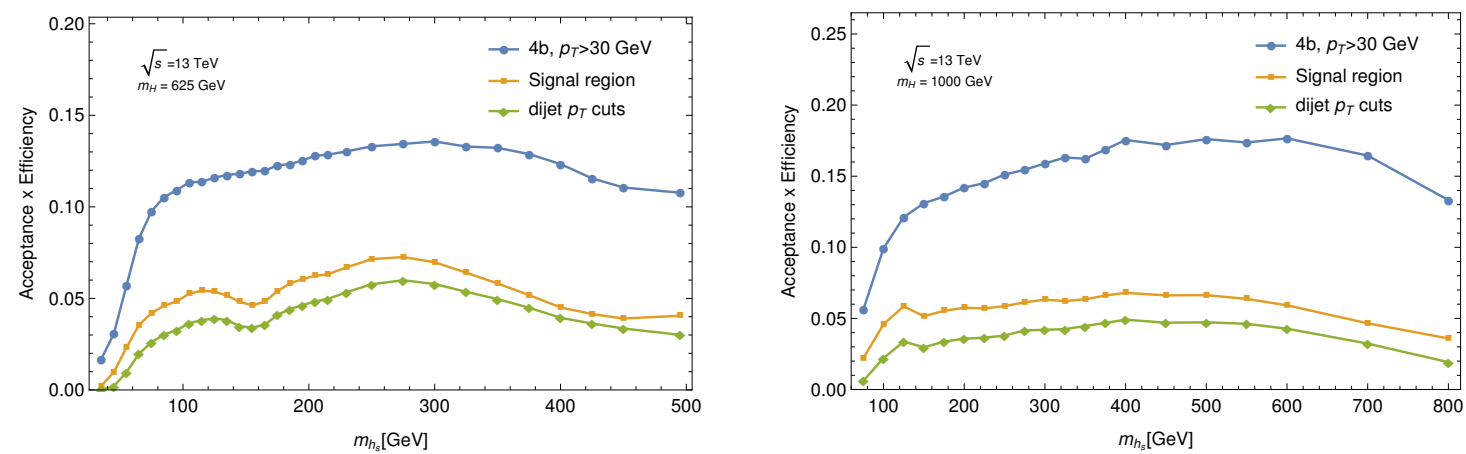

Figure 3. Efficiencies of the three cuts ( $4 b$ with $\left.p_{T}>30 \mathrm{GeV}\right)$, signal region (4.1) and dijet $p_{T}(4.2)$ for $M_{H}=625 \mathrm{GeV}$ and $M_{H}=1000 \mathrm{GeV}$ as function of $M_{H_{S}}$.

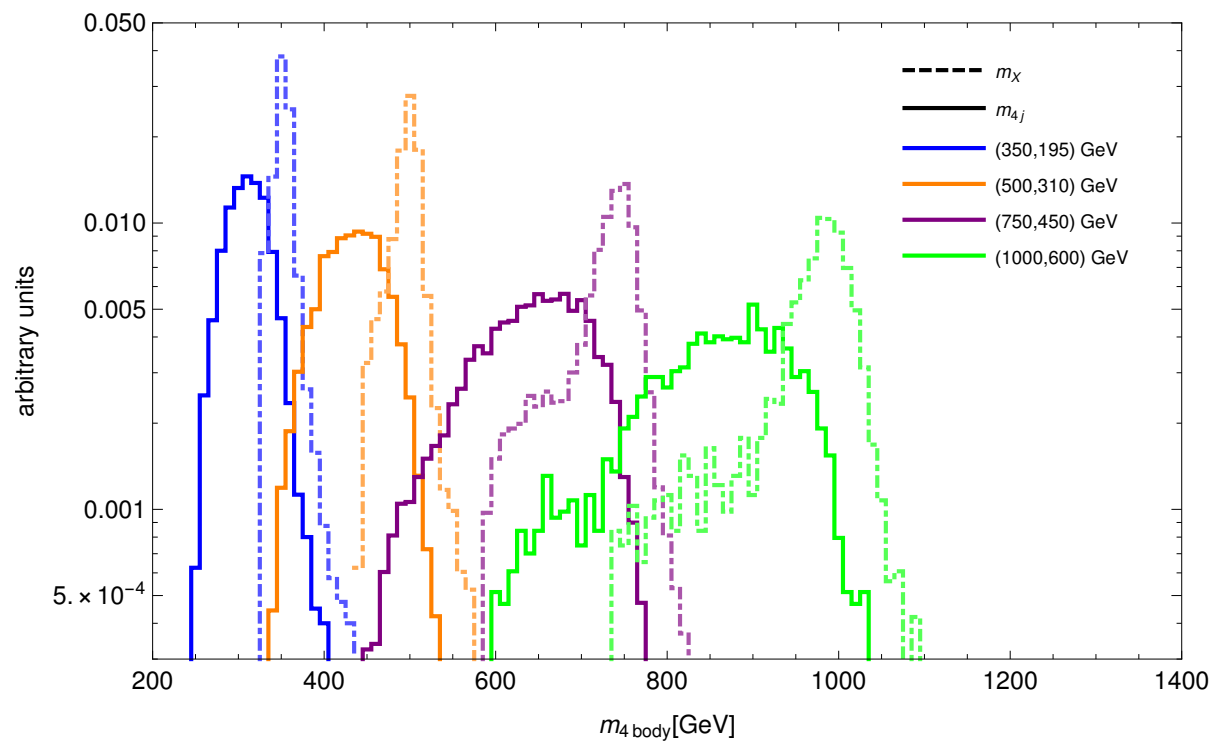

Figure 4. Distributions of the reconstructed masses $M_{4 b}$ (full lines) and $M_{X}$ (dashed lines) for the signal samples $\left(M_{H}, M_{H_{S}}\right)=(350,195),(500,310),(750,450),(1000,600) \mathrm{GeV}$.

In figure 4 we show the distributions of the reconstructed masses $M_{4 b}$ and $M_{X}$ for the signal samples $\left(M_{H}, M_{H_{S}}\right)=(350,195),(500,310),(750,450),(1000,600) \mathrm{GeV}$. We clearly see a sharpening of the peaks using $M_{X}$ in each case.

In order to obtain the required production cross sections times branching fractions for $5 \sigma$ discovery or $95 \%$ CL exclusion we have to obtain the background distribution of $M_{X}$.

\subsection{Background estimation}

Dominant backgrounds for the $b \bar{b} b \bar{b}$ final state are QCD multijet processes including jet misidentifications, and $t \bar{t}[6,14]$. The QCD multijet background is difficult to obtain from Monte Carlo simulations alone, and estimated from sidebands in [6, 14]. Such data is not available for the different values of $M_{H_{S}}$ studied here, with one exception: for $M_{H_{S}} \sim 125 \mathrm{GeV}$, the $b \bar{b} b \bar{b}$ final state coincides with the one searched for in [6, 14]. 
We simulated $b \bar{b} b \bar{b}, b \bar{b} c \bar{c}, b \bar{b} j j$ (with $j \neq c / \bar{c}$ ) and $t \bar{t}$ processes as we did for the signal samples in section 3. After applying the cuts of the previous subsection the relative contributions are $\sim 85-88 \%$ from $b \bar{b} b \bar{b}, \sim 7-8 \%$ from $b \bar{b} c \bar{c}, \sim 4-8 \%$ from $t \bar{t}$ (depending on $M_{H_{S}}$ and $M_{4 b}$ ). The $b \bar{b} j j$ contribution is only $0.8 \%$ and will subsequently be neglected. We checked that $b \bar{b} b \bar{b}+$ jets processes have little impact on the event shapes. Hence we did not simulate them separately, but took a NLO K-factor of 1.7 [37] into account.

In [6] the $M_{4 b}$ distribution of the multijet background has been obtained from a signal free sideband (with two $b$-tags only, and $M_{b \bar{b}}$ outside the search window) and appropriate rescaling using data with four $b$-tags, subtracting the $t \bar{t}$ contribution. The measured $M_{4 b}$ distribution is available in figure 5 in [6], where it is compared to the estimated background.

This allows us to proceed in a similar fashion: in order to compare to the data in [6], the previous cuts are slightly modified: the $b \bar{b}$ pairs are ordered in $p_{T}$ according to $M_{b \bar{b}}^{\text {lead }}$ and $M_{b \bar{b}}^{s u b l}$ and, as in [6], the signal region is defined by

$$
\chi=\sqrt{\left(\frac{M_{b \bar{b}}^{l e a d}-120 \mathrm{GeV}}{0.1 M_{b \bar{b}}^{l e a d}}\right)^{2}+\left(\frac{M_{b \bar{b}}^{s u b l}-0.85 M_{H_{S}}}{0.1 M_{b \bar{b}}^{s u b l}}\right)^{2}}<1.6 .
$$

Still (and expectedly) our simulated background falls below the measured data given in [6]. On the left hand side of figure 5 we show the $M_{4 b}$ distribution measured by [6] using $10.1 \mathrm{fb}^{-1}$ of integrated luminosity, and our MC result with statistical errors. The number of generated $\mathrm{MC}$ events corresponds to an equivalent integrated luminosity of $\sim 13 \mathrm{fb}^{-1}$. The statistical error per bin is thus obtained from the number of MC events per bin rescaled by $10 / 13$. The lower panels show the ratio $\mathrm{MC} /$ data bin by bin, with the uncertainties from the data and from our MC combined. At least for the interesting region $M_{4 b} \gtrsim 350 \mathrm{GeV}$ an overall rescaling of our multijet background, like it was performed in [6], seems appropriate. The rescaling factor is obtained taking the average of the data/MC ratio of all bins weighted by the corresponding uncertainties. We obtain a rescaling factor of $1.55 \pm 0.27$. (The $t \bar{t}$ background is left untouched, and remains at $\sim 3-6 \%$.) The comparison of the $M_{4 b}$ distribution of our background after rescaling to the data from [6] is shown on the right hand side of figure 5 .

Clearly it is somewhat optimistic to assume that the rescaling of the multijet background by $1.55 \pm 0.27$ remains valid for $M_{H_{S}} \neq 125 \mathrm{GeV}$. In the absence of data from sidebands this is, however, the best we can do. Subsequently \pm 0.27 will be used as an estimation of the systematic uncertainty of our background for all $M_{H_{S}}$, a number to be considered as indicative.

For forecasts at 300 or $3000 \mathrm{fb}^{-1}$ integrated luminosity the statistical uncertainties of the background are much smaller. It is then convenient to fit the shape of the $M_{X}$ background distributions (4.3) after cuts, which will be used in the following, by continuous functions. We found that the best fits are provided by a four parameter Gamma distribution defined in eq. (A.1) with $M_{H_{S}}$ dependent fit parameters. (The $b \bar{b} b \bar{b}, b \bar{b} c \bar{c}$, and $t \bar{t}$ background contributions to the $M_{X}$ distributions were fitted separately.) In figures 6 we show the sum of these fits for $M_{H_{S}}=85 \mathrm{GeV}$ and $M_{H_{S}}=350 \mathrm{GeV}$. 

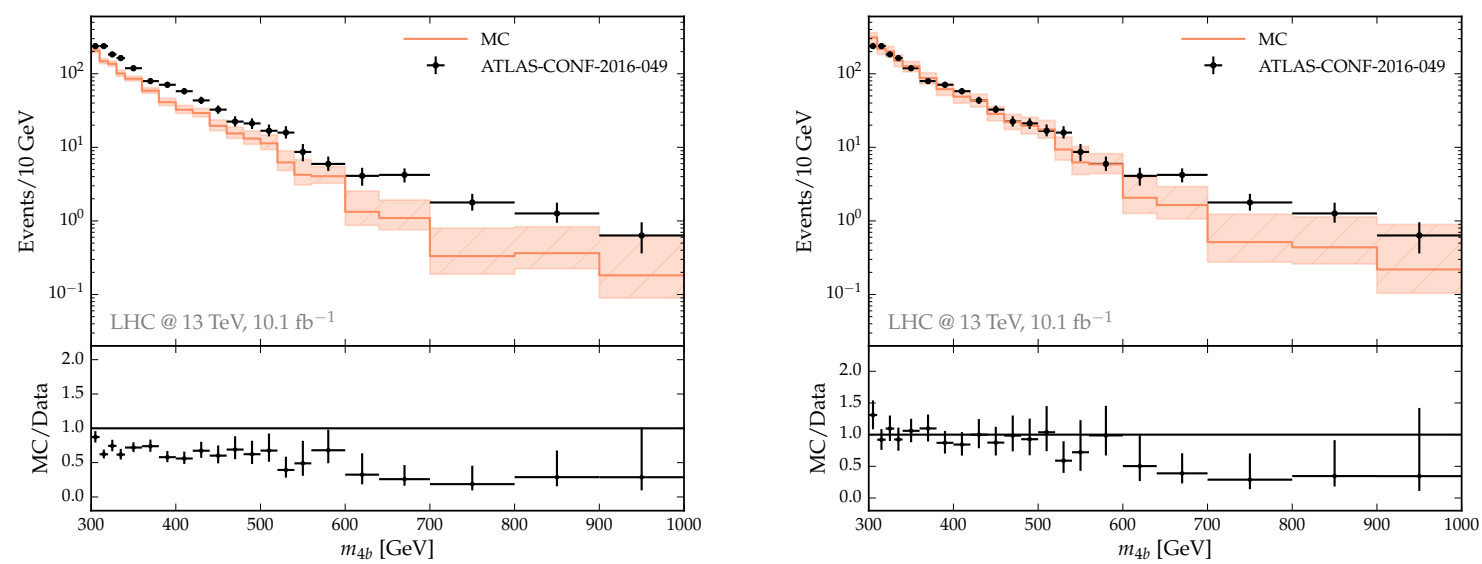

Figure 5. Left hand side: measured $M_{4 b}$ distribution from ATL-CONF-2016-049 [6], and our MC result for the background with statistical errors expected for $10.1 \mathrm{fb}^{-1}$ of integrated luminosity. The lower part shows the ratio $\mathrm{MC} /$ data bin by bin. Right hand side: the $M_{4 b}$ distribution of our background after rescaling, compared to the data in ATL-CONF-2016-049 [6].
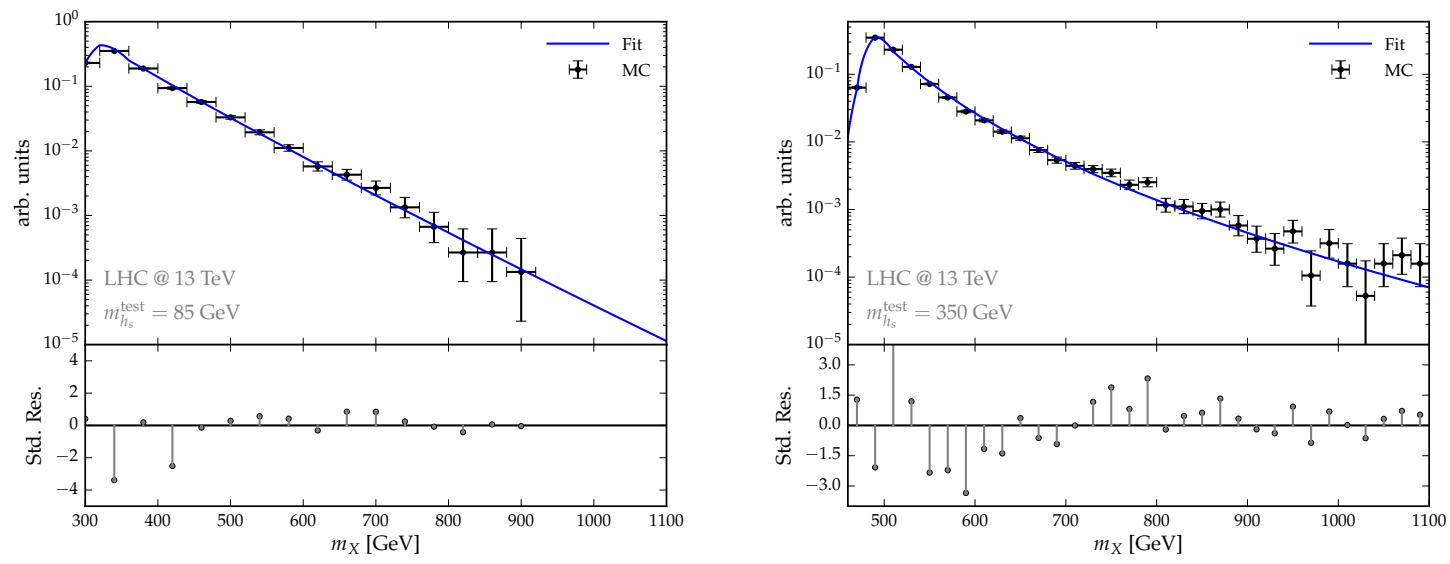

Figure 6. Fits by 4 parameter functions (A.1) to distributions of $M_{X}$ of the background obtained by Monte Carlo simulations of $b \bar{b} b \bar{b}, b \bar{b} c \bar{c}$ and $t \bar{t}$ events after cuts, for $M_{H_{S}}=85 \mathrm{GeV}$ (left) and $M_{H_{S}}=350 \mathrm{GeV}$ (right). The indicated uncertainties originate from the Monte Carlo samples.

Of course the remaining statistical fluctuations of the background can still be evaluated and combined with the systematic uncertainty (not shown in figures 6). It turns out, however, that for forecasts at 300 or $3000 \mathrm{fb}^{-1}$ the statistical fluctuations are negligibly small relative to the systematic uncertainty from the rescaling by $1.55 \pm 0.27$.

\subsection{Future $95 \%$ CL exclusion limits and $5 \sigma$ discovery cross sections}

Given the $M_{X}$ distribution of the background for various hypothetical values of $M_{H_{S}}$ and the $M_{X}$ distributions of signals one can, following the statistical methods from [52] and described in the appendix B, obtain values for 95\% CL exclusion limits and $5 \sigma$ discovery 


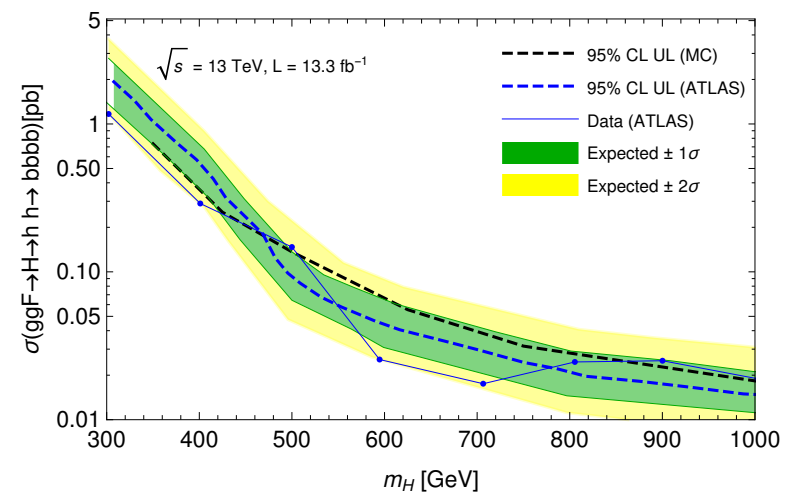

Figure 7. Expected 95\% CL upper limits from ATLAS [6] in blue, their $\pm 2 \sigma$ incertainty bands, the expected 95\% CL upper limits from our Monte Carlo in black and, for completeness, the 95\% CL upper limits obtained from the data.

limits for cross sections times branching fractions into the $b \bar{b} b \bar{b}$ final state as function of the integrated luminosity, $M_{H}$ and $M_{H_{S}}$.

In the case of an integrated luminosity of $13.3 \mathrm{fb}^{-1}$ at $13 \mathrm{TeV}$ we can compare the expected 95\% CL exclusion limits on cross sections times branching fractions to the ones given by ATLAS in figure 11 in [6], for $M_{X}=300 \ldots 1000 \mathrm{GeV}$ and $M_{H_{S}} \sim 125 \mathrm{GeV}$. (This ATLAS search was actually dedicated to spin 2 resonances decaying to SM Higgs pairs, but the differences to spin-0 resonances are expected to be small.) In figure 7 we show the expected 95\% CL upper limits from ATLAS, their $\pm 2 \sigma$ incertainty bands, the expected 95\% CL upper limits from our Monte Carlo and, for completeness, the 95\% CL upper limits obtained from the data. We see that our expected 95\% CL upper limits coincide well with the ones expected by ATLAS.

Since the background was fitted to data at $13 \mathrm{TeV}$ c.m. energy we will show our results also for $13 \mathrm{TeV}$, for 300 and $3000 \mathrm{fb}^{-1}$ integrated luminosity. We choose four representative values for $M_{H}=425,500,750$ and $1000 \mathrm{GeV}$, and show the $95 \% \mathrm{CL}$ exclusion limits and $5 \sigma$ discovery cross sections as function of $M_{H_{S}}$ in each case. For $300 \mathrm{fb}^{-1}$ integrated luminosity these are shown in figures 8 , for $3000 \mathrm{fb}^{-1}$ integrated luminosity in figures 9 .

The expected limits become weaker for $M_{H_{S}} \lesssim 50 \mathrm{GeV}$ (for $M_{X}=425-500 \mathrm{GeV}$ ) and $M_{H_{S}} \lesssim 100 \mathrm{GeV}$ (for $M_{H}=1000 \mathrm{GeV}$ ). As stated in subsection 4.1 here the $b \bar{b}$ pair from $H_{S}$ becomes too boosted and is no longer resolved by the standard jet clustering algorithm.

The shaded blue regions in figures 8 and 9 indicate viable values for the cross sections times branching fractions for $\sigma\left(g g F \rightarrow H \rightarrow H_{125}+H_{S} \rightarrow b \bar{b} b \bar{b}\right)$ in the parameter space of the NMSSM, see section 2. Typically the viable values for $\sigma\left(g g F \rightarrow A \rightarrow H_{125}+\right.$ $\left.A_{S} \rightarrow b \bar{b} b \bar{b}\right)$ are smaller; if not we show them as shaded red regions. In the region of the NMSSM parameter space corresponding to $M_{H} \gtrsim 500 \mathrm{GeV}$, the partial width for $H_{S} \rightarrow H_{125}+H_{125}$ becomes relatively large $(\approx 10 \mathrm{MeV})$ if kinematically allowed. As a consequence the branching fractions of $H_{S}$ into $b \bar{b}$ (and the other channels considered in this paper) decrease, leading to a decrease of the possible production cross sections times branching fractions for $M_{H} \gtrsim 500 \mathrm{GeV}, M_{H_{S}} \gtrsim 250 \mathrm{GeV}$. 

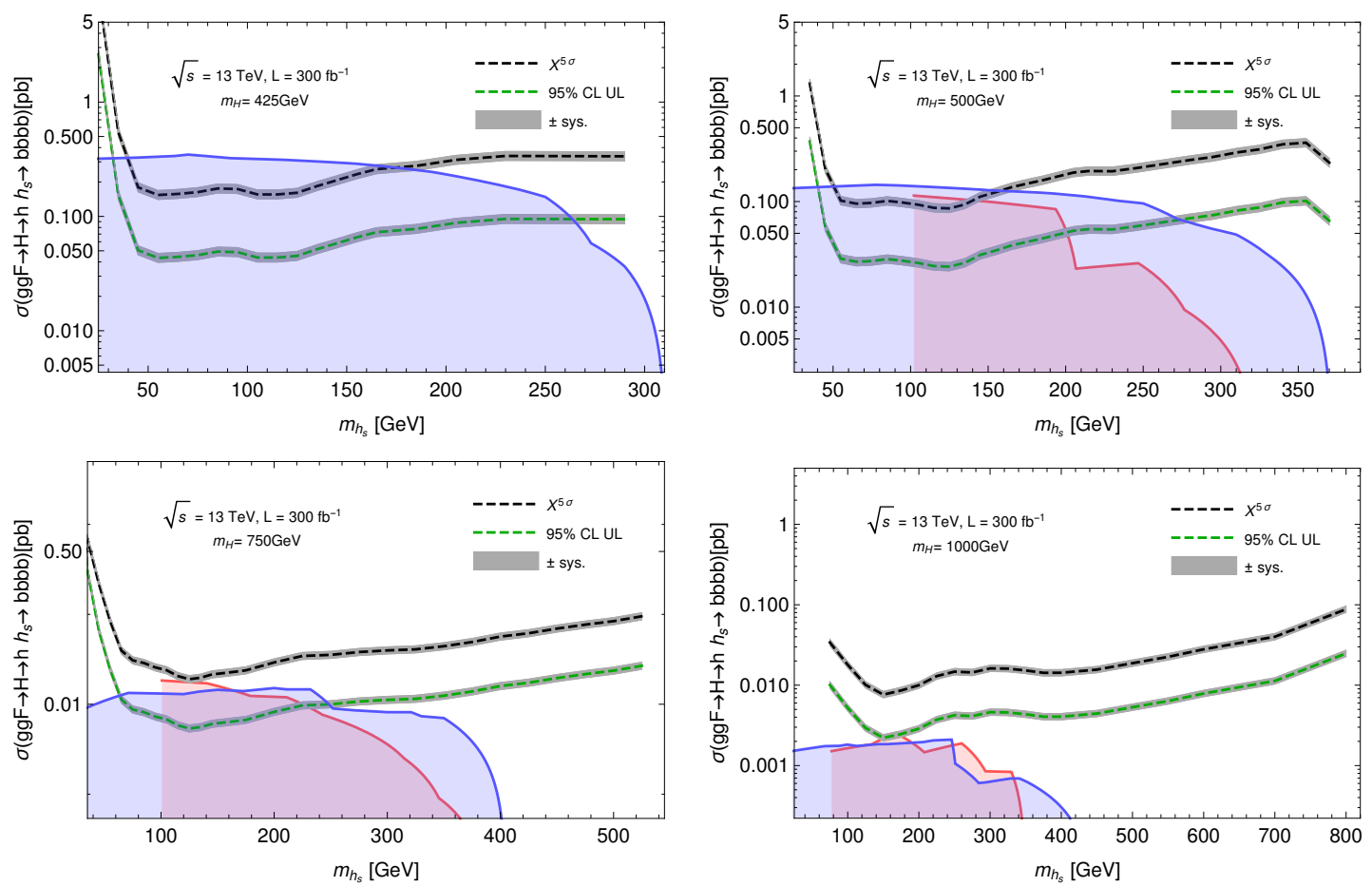

Figure 8. 95\% CL exclusion limits and $5 \sigma$ discovery cross sections in the $b \bar{b} b \bar{b}$ final state as function of $M_{H_{S}}$ for $300 \mathrm{fb}^{-1}$ integrated luminosity and $M_{H}=425 \mathrm{GeV}$ (upper left), $M_{H}=500 \mathrm{GeV}$ (upper right), $M_{H}=750 \mathrm{GeV}$ (lower left), $M_{H}=1000 \mathrm{GeV}$ (lower right). Shaded blue regions: viable values for $\sigma\left(g g F \rightarrow H \rightarrow H_{125}+H_{S} \rightarrow b \bar{b} b \bar{b}\right)$ in the parameter space of the NMSSM. Shaded red regions: viable values for $\sigma\left(g g F \rightarrow A \rightarrow H_{125}+A_{S} \rightarrow b \bar{b} b \bar{b}\right)$ in case they exceed potentially the ones for $\sigma\left(g g F \rightarrow H \rightarrow H_{125}+H_{S} \rightarrow b \bar{b} b \bar{b}\right)$.

The following conclusions can be drawn from figures 8 and 9: for $M_{H} \lesssim 500 \mathrm{GeV}$ wide ranges of $M_{H_{S}}$ in the NMSSM parameter space can be discovered or, at least, excluded. For larger $M_{H}$ testable regions in the NMSSM parameter space exist, but for $M_{H} \sim 1 \mathrm{TeV}$ only for $3000 \mathrm{fb}^{-1}$ integrated luminosity. In figure 10 we summarize these results showing the $95 \%$ C.L. expected upper limits in the $M_{H}$ vs $M_{H_{S}}$ plane.

We recall, however, that the sensitivities to cross sections in figures 8 and 9 are model independent and valid for arbitrary (e.g. non-supersymmetric) extensions of the Higgs sector.

\section{Search strategies for the $b \bar{b} \tau \tau$ final state}

Searches for resonant $H_{125}$ pair production in the $b \bar{b} \tau \tau$ final state have been performed by ATLAS at $8 \mathrm{TeV}$ [4], and by $\mathrm{CMS}$ at $13 \mathrm{TeV}$ in [16, 17, 19]. Following these searches we concentrate on the $\tau_{h} \tau_{h}, \tau_{h} \tau_{e}$ and $\tau_{h} \tau_{\mu}$ modes. As in the case of the $b \bar{b} b \bar{b}$ final state we optimise the cuts as function of a tentative value for $M_{H_{S}}$.

A priori the $\tau \tau$ pair can originate from $H_{S}$ or $H_{125}$; both cases will be studied below. For the analysis we will make no assumptions on the relative branching ratios $B R\left(H_{S} \rightarrow b \bar{b}\right)$ and $B R\left(H_{S} \rightarrow \tau \tau\right)$. The aim is to obtain separate $95 \% \mathrm{CL}$ exclusion limits and $5 \sigma$ 

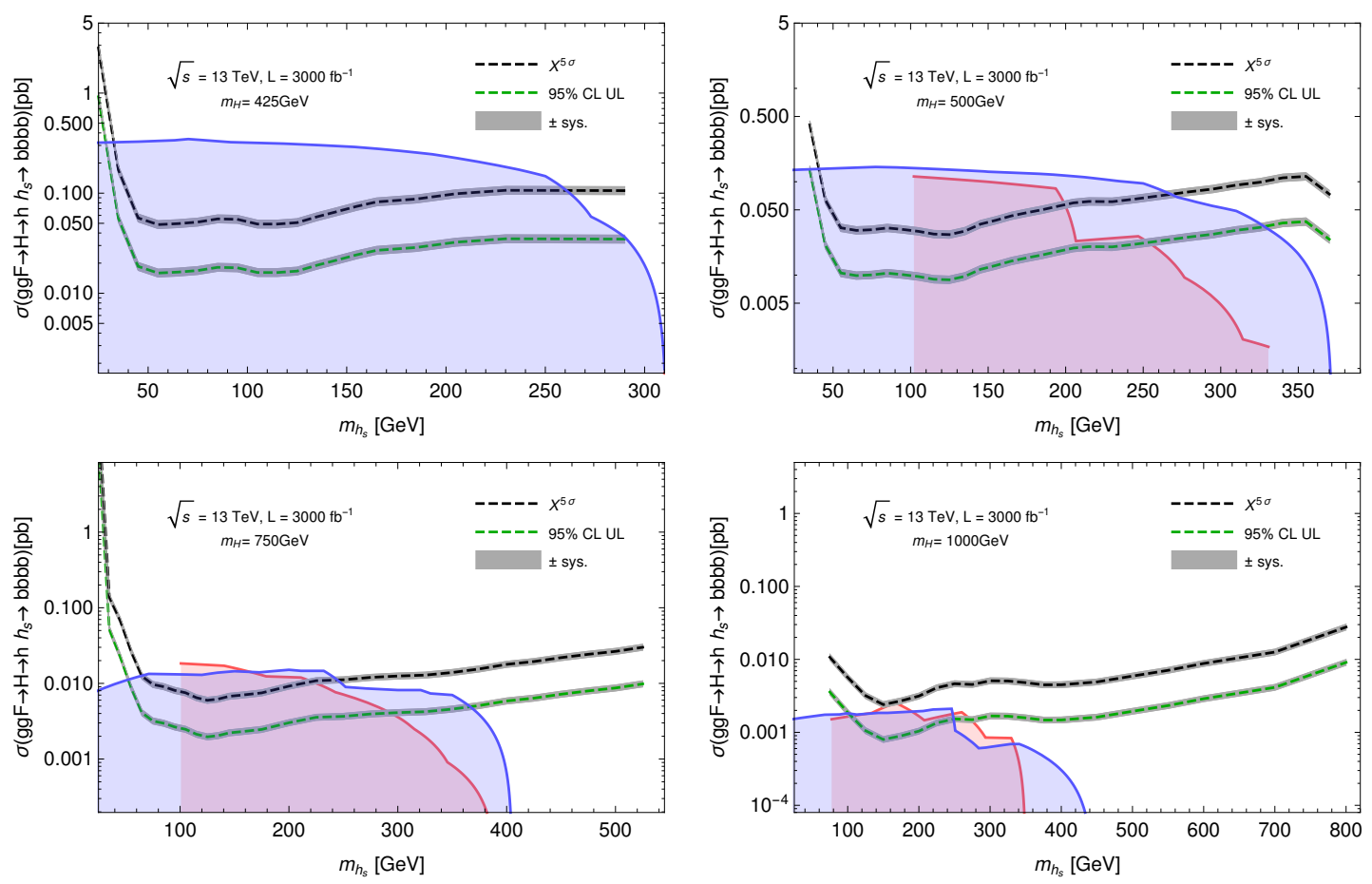

Figure 9. 95\% CL exclusion limits and $5 \sigma$ discovery cross sections in the $b \bar{b} b \bar{b}$ final state as function of $M_{H_{S}}$ for $3000 \mathrm{fb}^{-1}$ integrated luminosity and $M_{H}=425 \mathrm{GeV}$ (upper left), $M_{H}=500 \mathrm{GeV}$ (upper right), $M_{H}=750 \mathrm{GeV}$ (lower left), $M_{H}=1000 \mathrm{GeV}$ (lower right). The colored regions are explained in the caption of figure 8.

discovery cross sections for the processes $g g F \rightarrow H \rightarrow H_{S}(\rightarrow b \bar{b})+H_{125}(\rightarrow \tau \tau)$, and $g g F \rightarrow H \rightarrow H_{S}(\rightarrow \tau \tau)+H_{125}(\rightarrow b \bar{b})$.

\subsection{Analyses of signal samples}

For the simulation of signal samples the same series of codes as for the $b \bar{b} b \bar{b}$ final state was used, see section 3. Events are required to have exactly two $b$-tagged jets with $p_{T}(b)>$ $30 \mathrm{GeV}$ and $|\eta|<2.5$. For the $b$-tagging efficiency a working point with $\varepsilon_{b}=70 \%$ is chosen. If the event has exactly two hadronic taus $\tau_{h}$, both are required to have $p_{T}\left(\tau_{h}\right)>45 \mathrm{GeV}$. Events with one hadronic tau are required to have exactly one additional isolated lepton $\ell=e, \mu$ of opposite charge and with $p_{T}(\ell)>20 \mathrm{GeV}$. For the transverse mass $m_{T}^{\ell}$ of leptons we require

$$
m_{T}^{\ell} \equiv \sqrt{2 p_{T}(\ell) E_{T}^{\text {miss }}\left(1-\cos \left(\phi\left(E_{T}^{\text {miss }}\right)-\phi(\ell)\right)\right)}<40 \mathrm{GeV} .
$$

All objects are required to have $|\eta|<2.47$. In the case of $\tau_{h} \tau_{\ell}$ final states the invariant mass $M_{\tau \tau}$ was reconstructed using the collinear mass, i.e. assuming that the neutrino from the $\tau_{\ell}$ decay is emitted collinear to the lepton and responsible for all $E_{T}^{\text {miss }}$.

Considering first the case where the $\tau \tau$ pair originates from $H_{S}$ with an assumed mass $M_{H_{S}}$ (and hence that $M_{b \bar{b}}$ should be close to $125 \mathrm{GeV}$ ), a $M_{H_{S}}$ dependent signal region is 

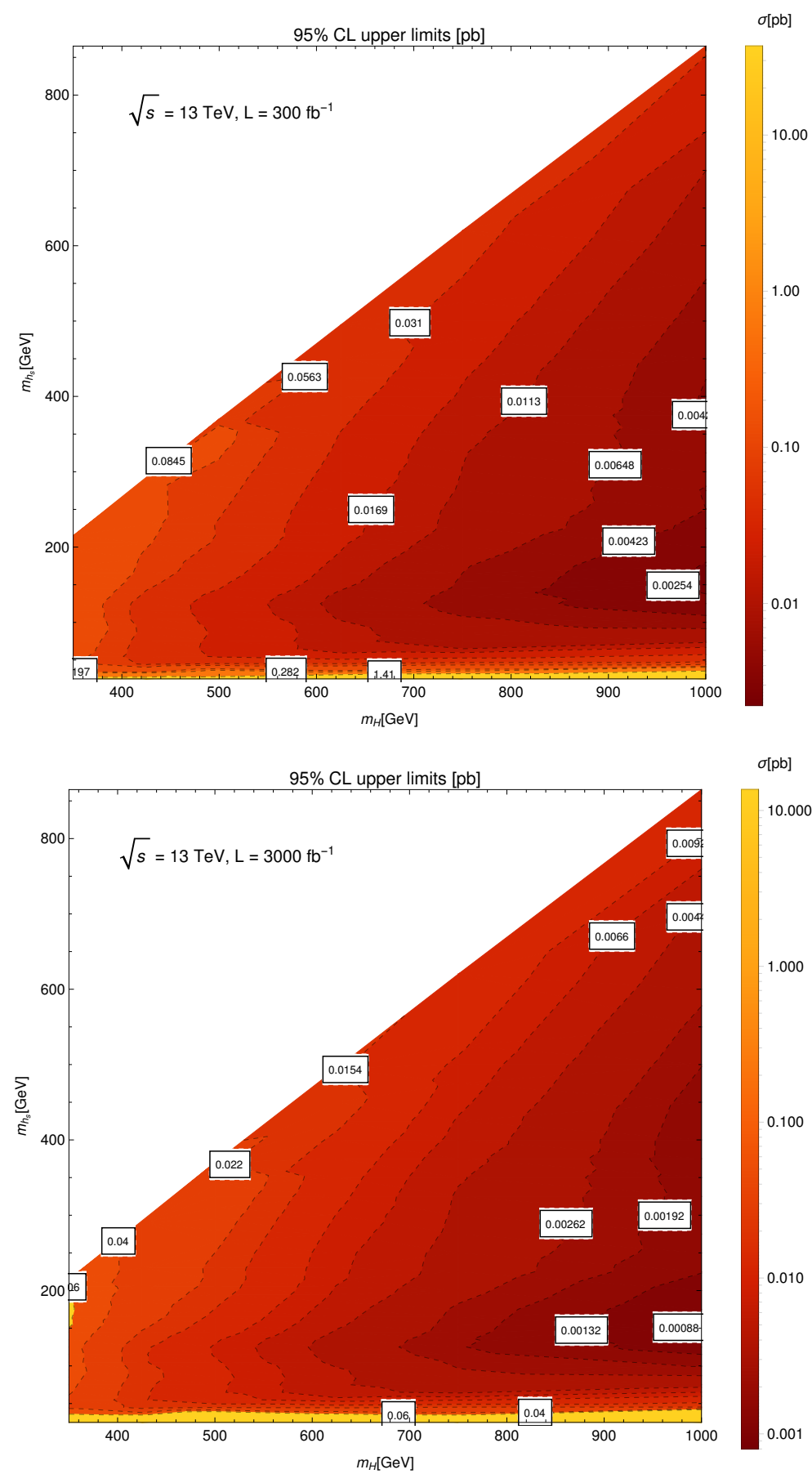

Figure 10. 95\% CL expected upper limits for the process $H \rightarrow H_{125} H_{S} \rightarrow b \bar{b} b \bar{b}$ for $\mathrm{L}=300 \mathrm{fb}^{-1}$ (end of Run III) (up) and $\mathrm{L}=3000 \mathrm{fb}^{-1}$ (HL-LHC) (down). 

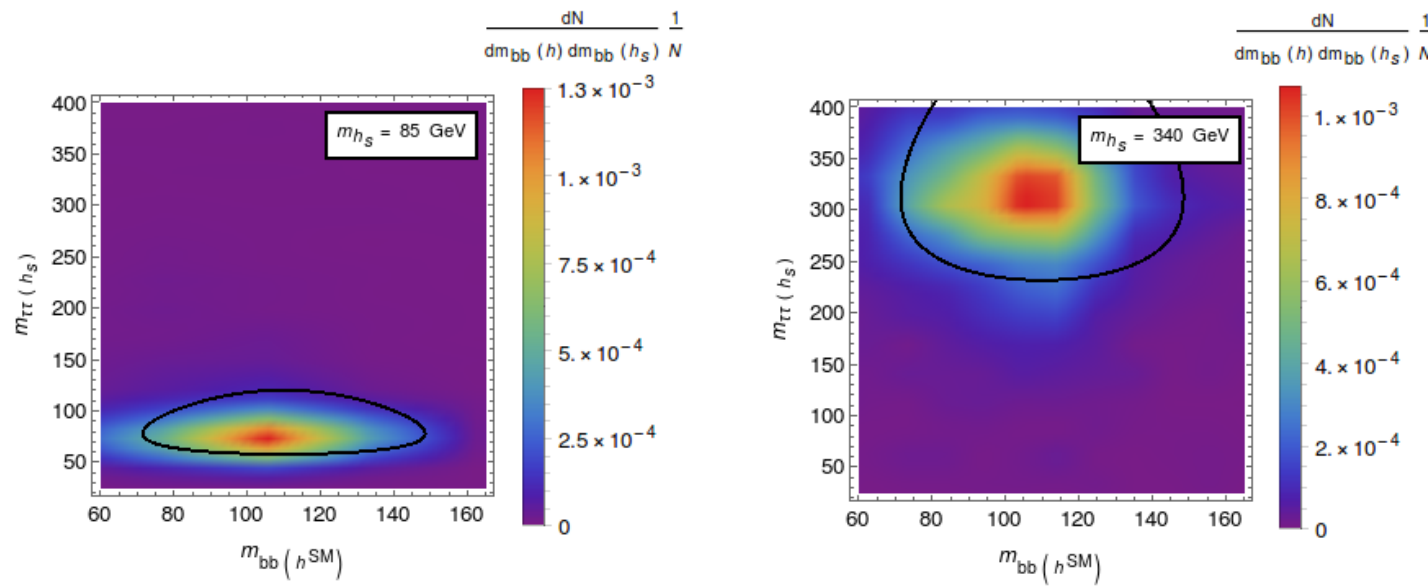

Figure 11. Dijet mass distributions $M_{b \bar{b}}\left(H_{125}\right)$ and $M_{\tau \tau}\left(H_{S}\right)$ for two different benchmark points $M_{H_{S}}=85 \mathrm{GeV}$ and $M_{H_{S}}=340 \mathrm{GeV}$. The black contours indicate the signal regions defined by $\chi<1$.

defined by

$$
\chi=\sqrt{\left(\frac{M_{b \bar{b}}-110 \mathrm{GeV}}{0.35 \cdot M_{b \bar{b}}}\right)^{2}+\left(\frac{M_{\tau \tau}-0.92 \cdot M_{H_{S}}}{\operatorname{Max}\left(0.35 \cdot M_{\tau \tau}, 30 \mathrm{GeV}\right)}\right)^{2}}<1 .
$$

If different pairings within a given event satisfy (5.2), the combination that minimizes $\chi$ is chosen. In figures 11 we show, for $M_{H}=500 \mathrm{GeV}$, the distributions of the dijet mass $M_{b \bar{b}}\left(H_{125}\right)$ and $M_{\tau \tau}\left(H_{S}\right)$ for the pairing minimizing $\chi$, for two different benchmark points $M_{H_{S}}=85 \mathrm{GeV}$ and $M_{H_{S}}=340 \mathrm{GeV}$ in the case where $M_{H_{S}}$ for the analysis was chosen correctly. The black contours indicate the signal regions defined by $\chi<1$.

Cuts on the transverse momenta of Higgs candidates are chosen as

$$
\begin{aligned}
& p_{T}(b \bar{b})>52 \mathrm{GeV}+0.14 M_{X}-0.2 M_{H_{S}}-\frac{M_{H_{S}}}{M_{X}} \cdot 202 \mathrm{GeV} \\
& p_{T}(\tau \tau)>24 \mathrm{GeV}+0.19 M_{X}-0.02 M_{H_{S}}-\frac{M_{H_{S}}}{M_{X}} \cdot 128 \mathrm{GeV}
\end{aligned}
$$

with

$$
M_{X}=M_{b \bar{b} \tau \tau}+125 \mathrm{GeV}-M_{b \bar{b}} .
$$

The numerical coefficients in eqs. (5.2)-(5.4) were obtained by optimizing the relative signal to background efficiency.

In the case where the $b \bar{b}$ pair originates from $H_{S}$ with an assumed mass $M_{H_{S}}$ (and hence that $M_{\tau \tau}$ should be close to $125 \mathrm{GeV}$ ), the signal region (5.2) is replaced by

$$
\chi=\sqrt{\left(\frac{M_{\tau \tau}-120 \mathrm{GeV}}{0.35 \cdot 120 \mathrm{GeV}}\right)^{2}+\left(\frac{M_{b \bar{b}}-0.85 \cdot M_{H_{S}}}{\operatorname{Max}\left(0.35 \cdot M_{H_{S}}, 35 \mathrm{GeV}\right)}\right)^{2}}<1
$$


and the cuts on the transverse momenta of Higgs candidates are

$$
\begin{aligned}
& p_{T}(\tau \tau)>118 \mathrm{GeV}+0.02 M_{X}-0.55 M_{H_{S}}-\frac{M_{H_{S}}}{M_{X}} \cdot 380 \mathrm{GeV} \\
& p_{T}(b \bar{b})>16 \mathrm{GeV}+0.19 M_{X}-0.02 M_{H_{S}}-\frac{M_{H_{S}}}{M_{X}} \cdot 137 \mathrm{GeV}
\end{aligned}
$$

with

$$
M_{X}=M_{b \bar{b} \tau \tau}+M_{H_{S}}-M_{b \bar{b}} .
$$

Hence, for each tentative value of $M_{H_{S}}$ two different analyses using different cuts are to be performed, resulting in two (slightly) different distributions of $M_{X}$.

\subsection{Background estimation}

Backgrounds originate from $t \bar{t}$ (and single top) and QCD+electroweak $b \bar{b} \tau \tau$ production. Contributions from one or more jets misidentified as $\tau_{h}$ are seen to become small after the cuts on $p_{T}(\tau \tau)$. We have generated $1.5 \cdot 10^{7} t \bar{t}$ events using MadGraph5_aMC@NLO [37]; the LO cross section was rescaled by a (NNLO+NNLL) K-factor 1.7 obtained from top++2.0 [4651]. MadGraph5_aMC@NLO was also used to generate QCD+electroweak $b \bar{b} \tau \tau$ events; the LO cross section was rescaled by a NLO K-factor 2.9 .

After applying the cuts of the previous subsection, the relative contributions of the SM backgrounds depend on $M_{H_{S}}$ and on whether the $\tau \tau$ pair originates from $H_{S}$ or $H_{125}$, although $t \bar{t}$ is always dominant: for $H_{S} \rightarrow \tau \tau$ the $t \bar{t}$ contribution increases from $\sim 60 \%$ for $M_{H_{S}} \sim 50 \mathrm{GeV}$ to $\sim 100 \%$ for $M_{H_{S}} \gtrsim 350 \mathrm{GeV}$, the remaining background stems from QCD+electroweak $b \bar{b} \tau \tau$ production. For $H_{125} \rightarrow \tau \tau$ the $t \bar{t}$ contribution is always $\sim 90 \%$.

In order to validate the background contribution to the $M_{X}$ distribution after cuts we use again a search for $H_{125}$ pair production at $13 \mathrm{TeV}$, now in the $b \bar{b} \tau \tau$ channel. Measurements of distributions of the (slightly corrected) total invariant mass by CMS, separately in the $b \bar{b} e \tau_{h}, b \bar{b} \mu \tau_{h}$ and $b \bar{b} \tau_{h} \tau_{h}$ channels, can be found in figure 1 in [17].

We have reproduced the cuts in [17] using our background samples. In figures 12 we show the measured total invariant mass distribution from figure 1 in [17] in black, and our MC results including the statistical uncertainties corresponding to $12.9 \mathrm{fb}^{-1}$ of integrated luminosity, in the three channels, in orange. Due to the smaller number of events the statistical uncertainties are now larger than in the $4 b$ case.

Still we can ask which rescaling of our simulated background, independent of the total invariant mass and common to all three channels (to improve the statistics), provides a best fit to the data. We find a factor $1.01 \pm 0.24$, and will subsequently use \pm 0.24 as an estimate of the systematic uncertainty of the background normalisation.

For forecasts at 300 or $3000 \mathrm{fb}^{-1}$ integrated luminosity the shape of the $M_{X}$ background distributions (5.4) will again be parametrized by continuous functions with $M_{H_{S}}$ dependent parameters: for the $t \bar{t}$ background the Frechet distribution, and for the $b \bar{b} \tau \tau$ background (all channels combined) the GaussExp function already used in [14]. Both functions are defined

in the appendix A. In figures 13 we show these fits for $M_{H_{S}}=85 \mathrm{GeV}$ and $M_{H_{S}}=350 \mathrm{GeV}$ where the $\tau \tau$ pair originates from $H_{S}$. 

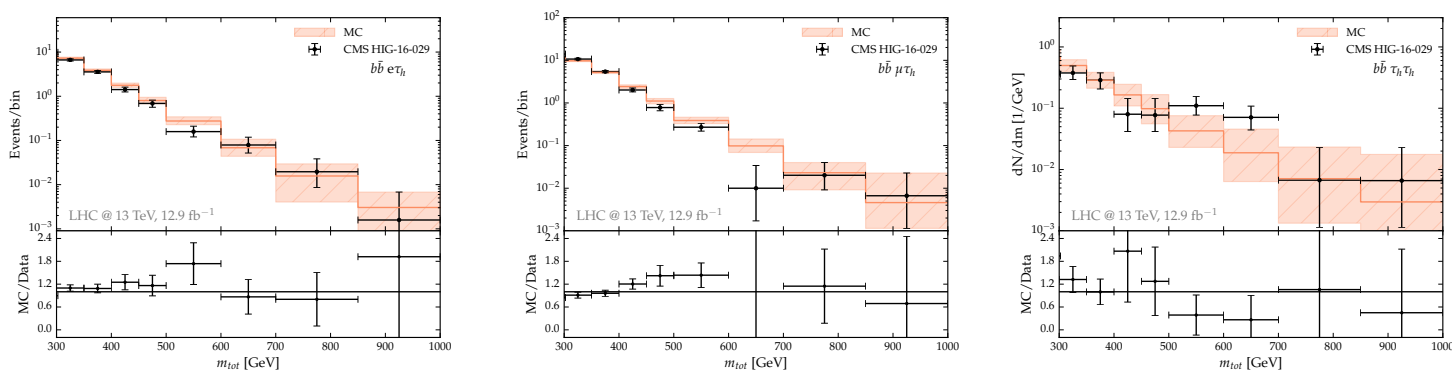

Figure 12. Measured total invariant mass distribution from [17] in black, and our MC results in orange including the statistical uncertainties corresponding to $12.9 \mathrm{fb}^{-1}$ of integrated luminosity in the three channels $b \bar{b} e \tau_{h}, b \bar{b} \mu \tau_{h}$ and $b \bar{b} \tau_{h} \tau_{h}$.
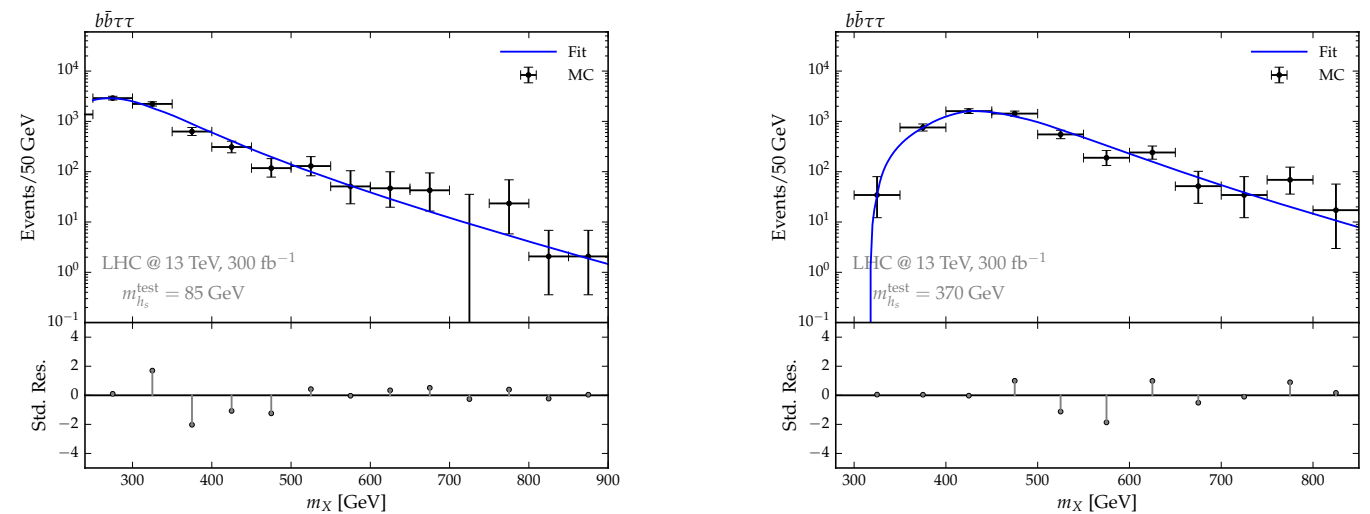

Figure 13. Fits to distributions of $M_{X}$ of the background obtained by Monte Carlo simulations of $t \bar{t}$ and $b \bar{b} \tau \tau$ events after cuts eq. (5.1)-(5.3) corresponding to $M_{H_{S}}=85 \mathrm{GeV}$ (left) and $M_{H_{S}}=$ $370 \mathrm{GeV}$ (right). The indicated uncertainties originate from the Monte Carlo samples.

\subsection{Future 95\% CL exclusion limits and $5 \sigma$ discovery cross sections}

Given the $M_{X}$ distribution of the background for various hypothetical values of $M_{H_{S}}$ and the $M_{X}$ distributions of signals we can, as before, obtain values for 95\% CL exclusion and $5 \sigma$ discovery for cross sections times branching fractions into the $H_{125} \rightarrow b \bar{b}, H_{S} \rightarrow \tau \tau$ and $H_{125} \rightarrow \tau \tau, H_{S} \rightarrow b \bar{b}$ final states as function of the integrated luminosity, $M_{H}$ and $M_{H_{S}}$. We choose four representative values for $M_{H}=425,500,750$ and $1000 \mathrm{GeV}$, and show the 95\% CL exclusion limits and $5 \sigma$ discovery cross sections as function of $M_{H_{S}}$ in each case. For $H_{125} \rightarrow b \bar{b}, H_{S} \rightarrow \tau \tau$ at $300 \mathrm{fb}^{-1}$ integrated luminosity these are shown in figures 14 , for $3000 \mathrm{fb}^{-1}$ integrated luminosity in figures 15. For $H_{125} \rightarrow \tau \tau, H_{S} \rightarrow b \bar{b}$ at $300 \mathrm{fb}^{-1}$ integrated luminosity these are shown in figures 16 , for $3000 \mathrm{fb}^{-1}$ integrated luminosity in figures 17. The uncertainties include statistical uncertainties and, added linearly, \pm 0.24 considered as an estimate of the systematic uncertainty originating from the normalisation of the background.

The following observations can be made: first, the expected sensitivities on cross sections times branching ratios differ hardly among the cases $H_{125} \rightarrow b \bar{b}$ and $H_{S} \rightarrow \tau \tau$ versus $H_{125} \rightarrow \tau \tau$ and $H_{S} \rightarrow b \bar{b}$; if at all, the analyses aiming at $H_{125} \rightarrow b \bar{b}$ and $H_{S} \rightarrow \tau \tau$ are typically somewhat more sensitive. 

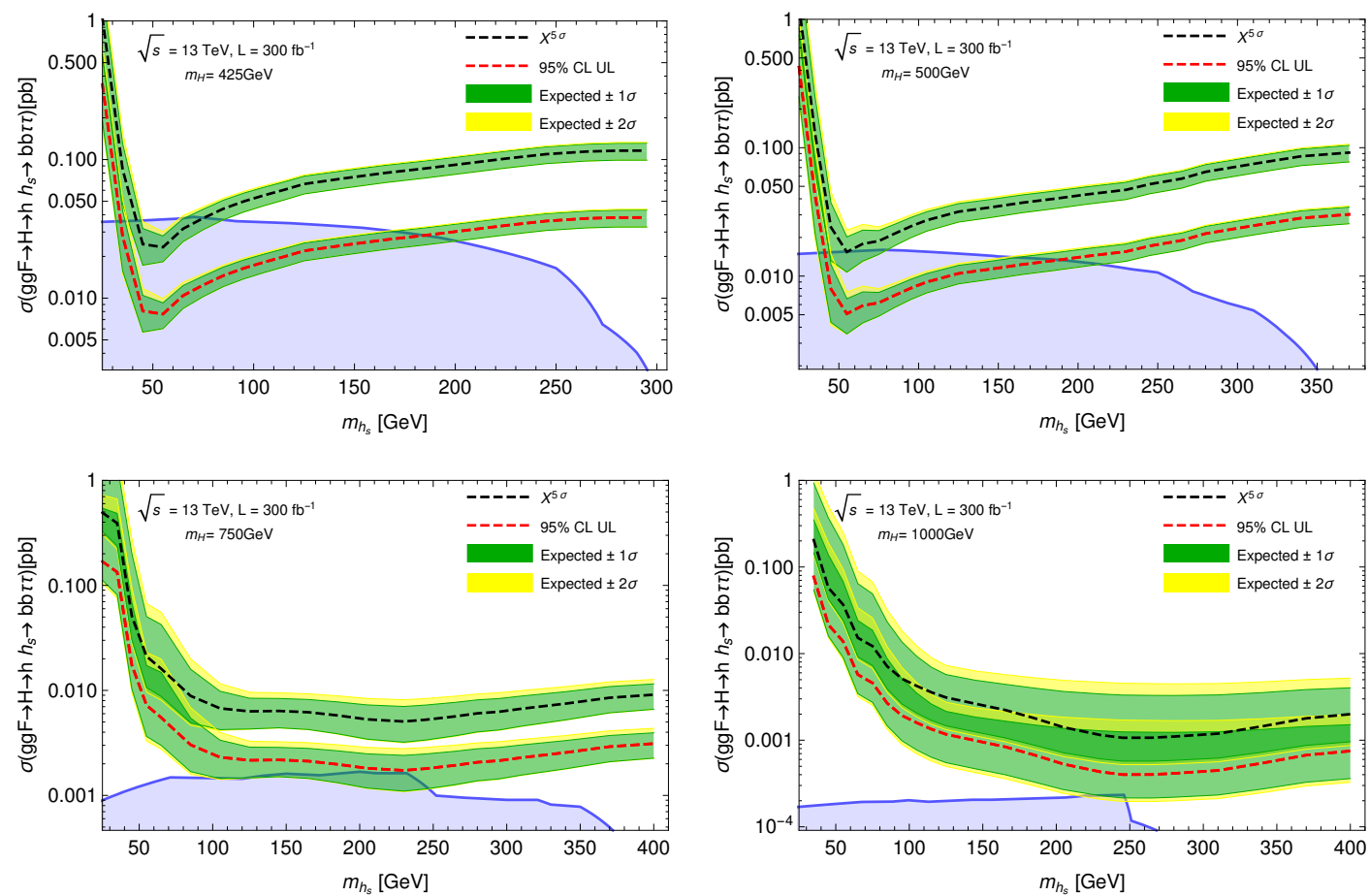

Figure 14. 95\% CL exclusion limits and $5 \sigma$ discovery cross sections for $H_{125} \rightarrow b \bar{b}$ and $H_{S} \rightarrow \tau \tau$ as function of $M_{H_{S}}$ for $300 \mathrm{fb}^{-1}$ integrated luminosity and $M_{H}=425 \mathrm{GeV}$ (upper left), $M_{H}=500 \mathrm{GeV}$ (upper right), $M_{H}=750 \mathrm{GeV}$ (lower left), $M_{H}=1000 \mathrm{GeV}$ (lower right).
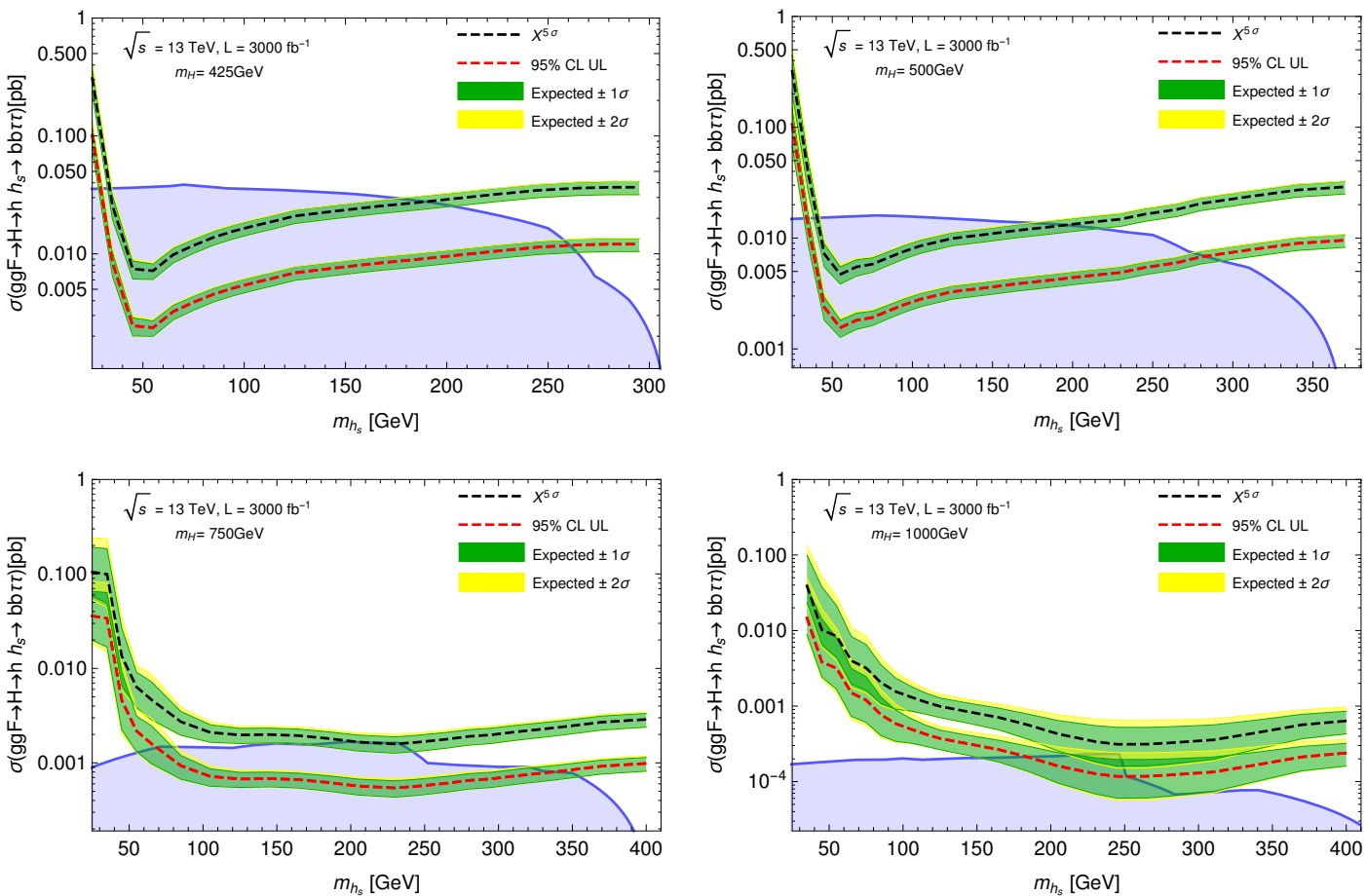

Figure 15. 95\% CL exclusion limits and $5 \sigma$ discovery cross sections for $H_{125} \rightarrow b \bar{b}$ and $H_{S} \rightarrow \tau \tau$ as function of $M_{H_{S}}$ for $3000 \mathrm{fb}^{-1}$ integrated luminosity and $M_{H}=425 \mathrm{GeV}$ (upper left), $M_{H}=$ $500 \mathrm{GeV}$ (upper right), $M_{H}=750 \mathrm{GeV}$ (lower left), $M_{H}=1000 \mathrm{GeV}$ (lower right). 

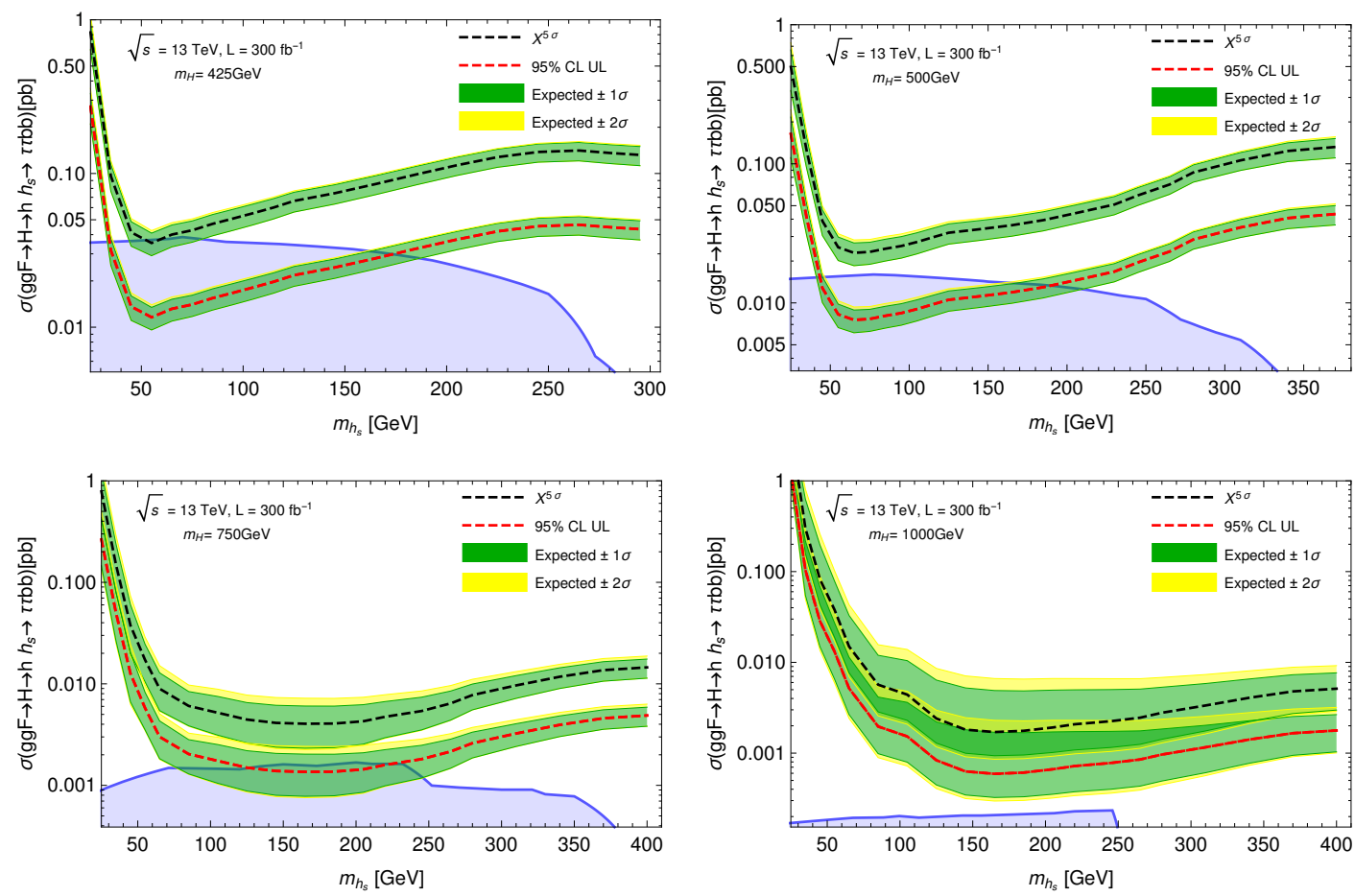

Figure 16. 95\% CL exclusion limits and $5 \sigma$ discovery cross sections for $H_{125} \rightarrow \tau \tau$ and $H_{S} \rightarrow b \bar{b}$ as function of $M_{H_{S}}$ for $300 \mathrm{fb}^{-1}$ integrated luminosity and $M_{H}=425 \mathrm{GeV}$ (upper left), $M_{H}=500 \mathrm{GeV}$ (upper right), $M_{H}=750 \mathrm{GeV}$ (lower left), $M_{H}=1000 \mathrm{GeV}$ (lower right).
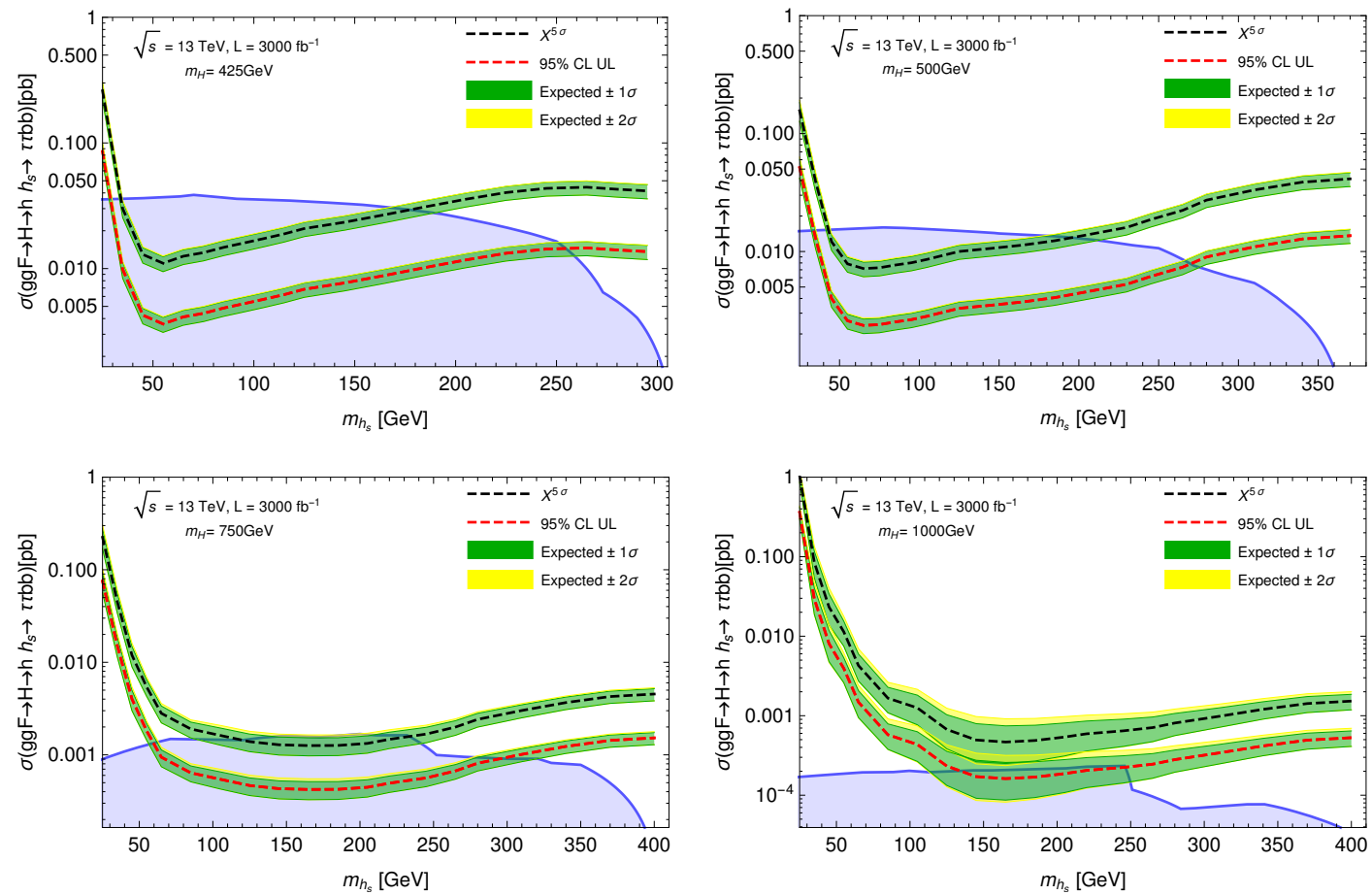

Figure 17. 95\% CL exclusion limits and $5 \sigma$ discovery cross sections for $H_{125} \rightarrow \tau \tau$ and $H_{S} \rightarrow b \bar{b}$ as function of $M_{H_{S}}$ for $3000 \mathrm{fb}^{-1}$ integrated luminosity and $M_{H}=425 \mathrm{GeV}$ (upper left), $M_{H}=$ $500 \mathrm{GeV}$ (upper right), $M_{H}=750 \mathrm{GeV}$ (lower left), $M_{H}=1000 \mathrm{GeV}$ (lower right). 
Second, in Two-Higgs-Doublet models of type II as well as in the NMSSM the branching fractions into $b \bar{b}$ and $\tau \tau$ of both $H_{125}$ and $H_{S}$ are always related by a factor $\sim 9: 1$. Accordingly the possible cross sections times branching fractions in the NMSSM parameter space for both $\sigma\left(g g F \rightarrow H \rightarrow H_{125}+H_{S} \rightarrow b \bar{b} \tau \tau\right)$ and $\sigma\left(g g F \rightarrow H \rightarrow H_{125}+H_{S} \rightarrow \tau \tau b \bar{b}\right)$, indicated in blue in figures 14-17, are $\sim 1 / 9$ of the ones in figures 8-9 for the $b \bar{b} b \bar{b}$ final state. (The same reasoning applies to $\sigma\left(g g F \rightarrow A \rightarrow H_{125}+A_{S} \rightarrow b \bar{b} \tau \tau\right)$ and $\sigma(g g F \rightarrow$ $\left.A \rightarrow H_{125}+A_{S} \rightarrow \tau \tau b \bar{b}\right)$; the viable NMSSM points correspond to the ones in figures 8 and 9.)

Then one can ask, for a given point in parameter space, which of the analyses considered up to now is the most sensitive. According to our results this is the search in the $b \bar{b} b \bar{b}$ final state which allows to test somewhat larger regions in parameter space.

\section{Search strategies for the $b \bar{b} \gamma \gamma$ final state}

Searches for resonant $H_{125}$ pair production in the $b \bar{b} \gamma \gamma$ final state have been performed by ATLAS at $8 \mathrm{TeV}[2,4]$ and at $13 \mathrm{TeV}[5]$, by $\mathrm{CMS}$ at $8 \mathrm{TeV}$ in [7] and at $13 \mathrm{TeV}$ in [18, 21]. A priori the diphotons can originate from $H_{S}$ or $H_{125}$; both cases will be studied below. As in case of the previous final states we optimise the cuts as function of a tentative value for $M_{H_{S}}$.

\subsection{Analyses of signal samples}

For the simulation of signal samples we used again MadGraph5_aMC@NLO [37]. Events are required to have exactly two $b$-tagged jets with $p_{T}(b)>40 \mathrm{GeV}$ and $|\eta|<2.5$. Following ATLAS [5] a working point with $\epsilon_{b}=0.85$ was chosen for the $b$-tagging efficiency in order to increase the statistics.

At least two photons are required in each event which have to satisfy the isolation criteria

$$
\frac{\sum_{i} p_{T, i}}{p_{T, \gamma}}<0.1
$$

where the sum over $i$ includes all tracks within a cone $\Delta R=0.4$ around the photon. The two leading photons are required to satisfy

$$
E_{T}^{\text {lead }} / M_{\gamma \gamma}>0.35, \quad E_{T}^{\text {subl }} / M_{\gamma \gamma}>0.25, \quad|\eta|<2.37 .
$$

Additional cuts depend on whether the diphoton pair is assumed to originate from $H_{125}$ or $H_{S}$, and the assumed value of $M_{H_{S}}$. First we consider the case $H_{S} \rightarrow \gamma \gamma$. Then the $b \bar{b}$ pair is assumed to originate from $H_{125}$, and we require

$$
100 \mathrm{GeV}<M_{b \bar{b}}<150 \mathrm{GeV}
$$

As in the previous cases it is useful to define a corrected invariant mass $M_{X}$ for the $b \bar{b} \gamma \gamma$ system:

$$
M_{X}=M_{b \bar{b} \gamma \gamma}+125 \mathrm{GeV}-M_{b \bar{b}}
$$


Given an assumed value for $M_{H_{S}}$ it turned out to optimize the signal to background efficiency applying a $M_{X}$ and $M_{H_{S}}$ dependent cut on the diphoton invariant mass $M_{\gamma \gamma}$, since the measured distribution $\left|M_{\gamma \gamma}-M_{H_{S}}\right|$ broadens somewhat with $M_{X}$ :

$$
\left|M_{\gamma \gamma}-M_{H_{S}}\right|<4.3 \mathrm{GeV}+0.016 M_{X} .
$$

Also the cuts on the $H_{125}$ and $H_{S}$ candidates depend on $M_{X}$ :

$$
\begin{aligned}
p_{T}(b \bar{b}) & >17 \mathrm{GeV}+0.18 M_{X}, \\
E_{T}(\gamma \gamma) & =68.3 \mathrm{GeV}+0.25 M_{X} .
\end{aligned}
$$

( $E_{T}(\gamma \gamma)$ instead of $p_{T}(\gamma \gamma)$ allows for $M_{H_{S}}$ independent cuts.)

Assuming $H_{125} \rightarrow \gamma \gamma$ and $H_{S} \rightarrow b \bar{b}$ the previous cuts are modified as follows: first, for $M_{X}$ we take

$$
M_{X}=M_{b \bar{b} \gamma \gamma}+M_{H_{S}}-M_{b \bar{b}}
$$

and for the diphoton invariant mass we require

$$
\left|M_{\gamma \gamma}-125 \mathrm{GeV}\right|<2 \mathrm{GeV}+0.02 M_{X} .
$$

The mass window for $M_{b \bar{b}}$ is now

$$
0.9 M_{H_{S}}-30 \mathrm{GeV}<M_{b \bar{b}}<0.9 M_{H_{S}}+20 \mathrm{GeV} .
$$

The cuts on the $H_{125}$ and $H_{S}$ candidates are:

$$
\begin{aligned}
p_{T}(b \bar{b}) & >-5.7 \mathrm{GeV}+0.29 M_{X}, \\
E_{T}(\gamma \gamma) & =7.45 \mathrm{GeV}+0.33 M_{X} .
\end{aligned}
$$

All the numerical values above have been obtained by optimising signal-to-background ratios using the $\mathrm{MC}$ events.

\subsection{Background estimation}

SM backgrounds originate from $b \bar{b} \gamma \gamma, c \bar{c} \gamma \gamma, j j \gamma \gamma(j \neq c / \bar{c}), b \bar{b} j \gamma$ and $t \bar{t} H$. We simulated these backgrounds again using MadGraph5_aMC@NLO. Due to the relatively large $b$-tagging efficiency $\epsilon=0.85$ mistagging rates are relatively large. $b \bar{b} j \gamma$ contribute if another fake photon appears.

After applying the cuts of the previous subsection the relative contributions of the SM backgrounds depend strongly on $M_{H_{S}}$ and $M_{H}$, and on whether the $\gamma \gamma$ pair originates from $H_{S}$ or $H_{125}$. For a light $H_{S} \sim 85 \mathrm{GeV}$ the background from $b \bar{b} j \gamma$ is always important, $\sim 60 \%$ for $H_{S} \rightarrow \gamma \gamma$ and $\sim 30 \%$ for $H_{S} \rightarrow b \bar{b}$. However, all other $\gamma \gamma+X$ SM backgrounds can also contribute several $10 \%$ individually. The contribution from $t \bar{t} H$ is always $\lesssim 3 \%$.

For $b \bar{b} \gamma \gamma, c \bar{c} \gamma \gamma$ and $j j \gamma \gamma$ an extra jet was allowed in the final state, $b \bar{b} j \gamma$ was multiplied by a NLO K factor 1.58 from [37], and $t \bar{t} H$ (with SM couplings) was simulated at NLO.

Still we cannot expect that the total SM background cross section is completely captured by the MC simulation; in searches by ATLAS and CMS in [5, 18, 21] sidebands are 
used for this purpose. Thus we proceed as before and correct the total background cross section using data driven methods in the particular case $M_{H_{S}} \sim 125 \mathrm{GeV}$ equivalent to (resonant) SM Higgs pair production. To this end we modify slightly the cuts on $p_{T}(b)$ ( $>55 \mathrm{GeV}$ and $>35 \mathrm{GeV}$ for the leading and next-to-leading $b$-jets), $M_{b \bar{b}}$ and $M_{\gamma \gamma}$ such that they coincide with the ones in the ATLAS search [5]. (In the case of $M_{\gamma \gamma}$ we could check that the efficiency coincides.) Extrapolating from sidebands with less $b$-jets, ATLAS [5] obtained $1.63 \pm 0.3$ expected background events in the signal region, whereas we found $1.06 \pm 0.14$ events from summing all MC events. Since all our backgrounds were simulated to similar order (NLO) in the QCD coupling and separate higher order K factors are not available, we multiply their sum by $1.54 \pm 0.35$ where the latter uncertainty will again be treated as an estimate of the systematic uncertainty contributing to our final results.

The dependence of the background on the total invariant mass $M_{b \bar{b} \gamma \gamma}$ was parametrized in [5] by a two parameter Landau distribution given in appendix A. We found that the Landau distribution fits the $M_{X}$ distribution from eq. (6.8) as well (with $M_{H_{S}}$ dependent parameters), and used it for the expected $M_{X}$ distributions of the various background contributions for our forecasts at $300 \mathrm{fb}^{-1}$ and $3000 \mathrm{fb}^{-1}$.

\subsection{Future 95\% CL exclusion limits and $5 \sigma$ discovery cross sections}

Given the $M_{X}$ distribution of the background for various hypothetical values of $M_{H_{S}}$ and the $M_{X}$ distributions of signals we can, as before, obtain values for 95\% CL exclusion and $5 \sigma$ discovery for cross sections times branching fractions into the $H_{125} \rightarrow b \bar{b}, H_{S} \rightarrow \gamma \gamma$ and $H_{125} \rightarrow \gamma \gamma, H_{S} \rightarrow b \bar{b}$ final states as function of the integrated luminosity, $M_{H}$ and $M_{H_{S}}$.

After completing our analysis the CMS search [21] for resonant Higgs pair production in the channel $H_{125}+H_{125} \rightarrow b \bar{b} \gamma \gamma$ based on $35.9 \mathrm{fb}^{-1}$ appeared. The expected $95 \%$ CL exclusion limits given in [21] can be compared to ours for $M_{H_{S}}=125 \mathrm{GeV}$ for the same integrated luminosity; this comparison as function of $M_{H}$ is shown in figure 18 . The expected limits coincide within $1 \sigma$ for $M_{H} \gtrsim 500 \mathrm{GeV}$, and within $2 \sigma$ everywhere. Our expected limits are systematically more conservative; we note that the CMS analysis employs a trained boosted decision tree in order to separate the signal from the backgrounds which is not available here.

Our expected 95\% CL exclusion limits and $5 \sigma$ discovery cross sections for $H_{125} \rightarrow b \bar{b}$, $H_{S} \rightarrow \gamma \gamma$ as function of $M_{H_{S}}$ at $300 \mathrm{fb}^{-1}$ integrated luminosity are shown in figures 19 for four representative values for $M_{H}=425,500,625$ and $750 \mathrm{GeV}$, and for $3000 \mathrm{fb}^{-1}$ integrated luminosity in figures 20. For $H_{125} \rightarrow \gamma \gamma, H_{S} \rightarrow b \bar{b}$ at $300 \mathrm{fb}^{-1}$ integrated luminosity these are shown in figures 21 , for $3000 \mathrm{fb}^{-1}$ integrated luminosity in figures 22 . As in the $b \bar{b} b \bar{b}$ case, we also present the results for the expected 95\% C.L. upper limits in the $M_{H_{125}}$ vs $M_{H_{S}}$ plane in figure 23 .

The uncertainties include statistical uncertainties and, added linearly, a factor \pm 0.35 considered as systematic uncertainty originating from the normalisation of the background.

As before viable NMSSM regions for scalar production are shown in shaded blue, for pseudoscalar production in shaded red in case they potentially exceed the ones for scalar production. Again a sizeable region in the NMSSM parameter space can be tested in this final state provided $M_{H}$ is not too large. It is, however, not the same region 


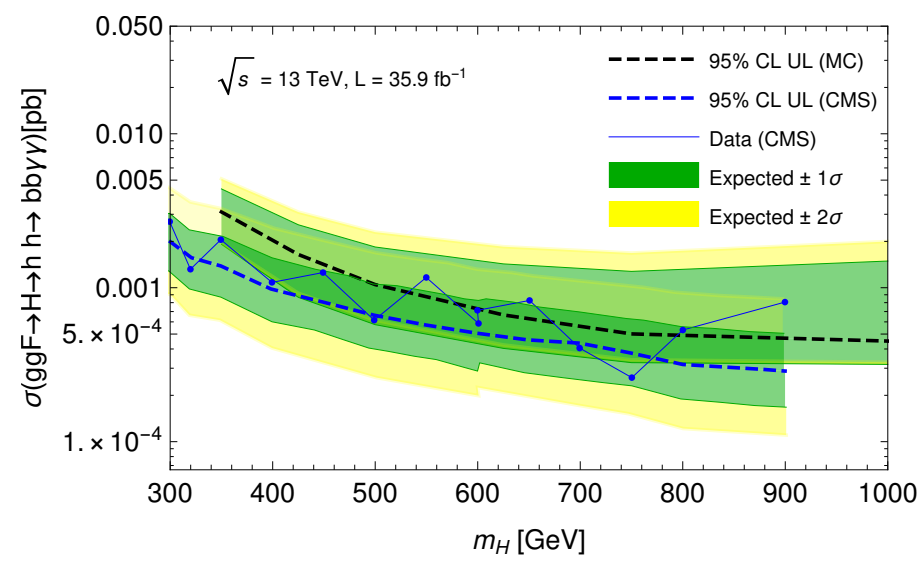

Figure 18. Expected 95\% CL exclusion limits for $g g F \rightarrow H_{125} H_{125} \rightarrow b \bar{b} \gamma \gamma$ for $35.9 \mathrm{fb}^{-1}$ from CMS [21] and from our MC simulation as function of $M_{H}$. For completeness the observed limits are shown.
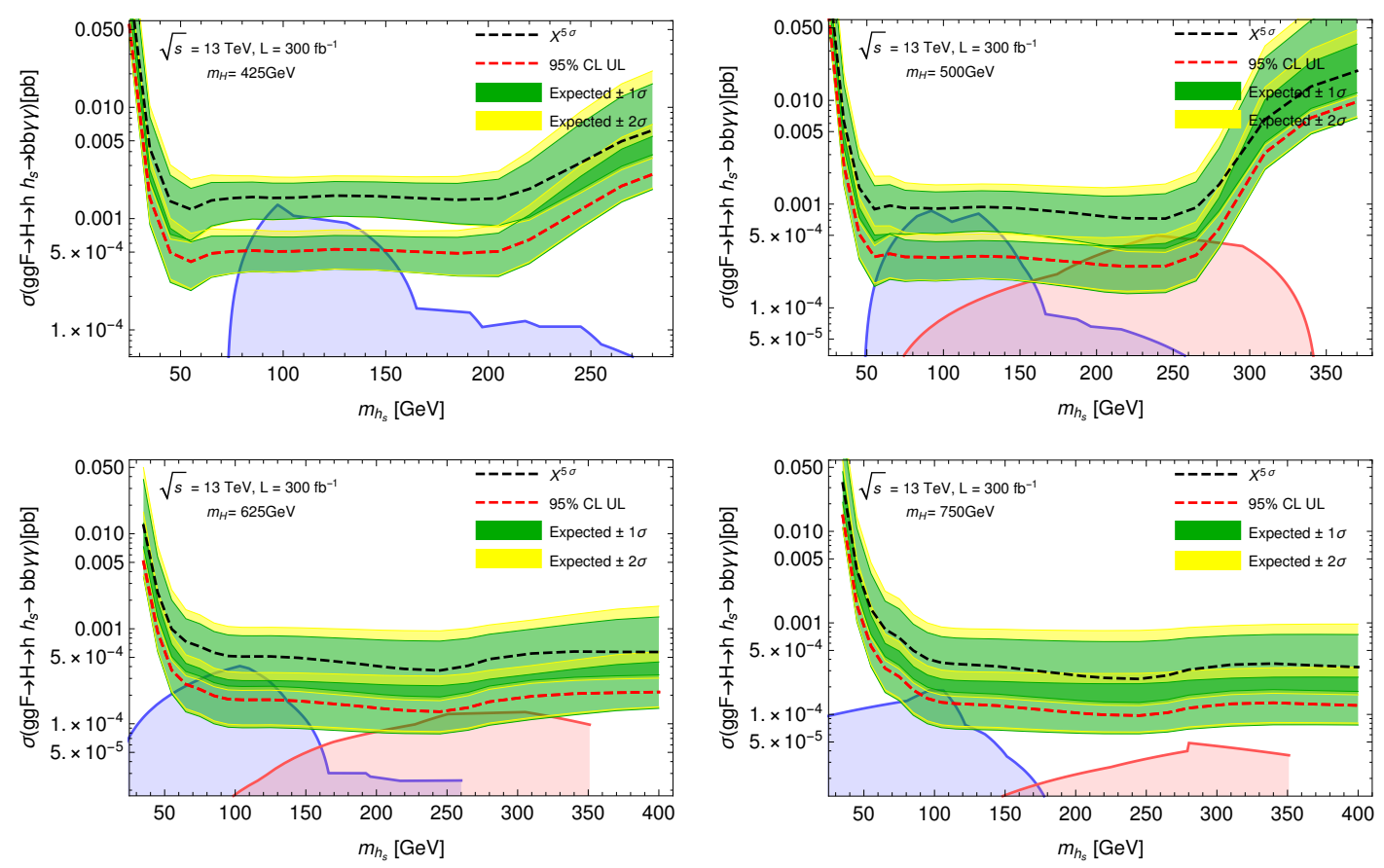

Figure 19. 95\% CL exclusion limits and $5 \sigma$ discovery cross sections for $H_{125} \rightarrow b \bar{b}$ and $H_{S} \rightarrow \gamma \gamma$ as function of $M_{H_{S}}$ for $300 \mathrm{fb}^{-1}$ integrated luminosity and $M_{H}=425 \mathrm{GeV}$ (upper left), $M_{H}=500 \mathrm{GeV}$ (upper right), $M_{H}=625 \mathrm{GeV}$ (lower left), $M_{H}=750 \mathrm{GeV}$ (lower right). The colored regions are explained in the caption of figure 8.

potentially visible in the $b \bar{b} b \bar{b}$ final state: the branching fraction of $H_{S}$ into $\gamma \gamma$ can vary in the $0.2 \% \pm 0.1 \%$ range, and is anticorrelated with its branching fraction into $b \bar{b}$. Moreover, as it is visible from the shaded red regions in figures 19 and 20, the signal rates $\sigma(g g F \rightarrow$ $\left.A \rightarrow H_{125}+A_{S} \rightarrow b \bar{b} \gamma \gamma\right)$ can be relatively large. These correspond to very singletlike pseudoscalars $A_{S}$ with very suppressed couplings to quarks and leptons, but sizeable coupling $\sim \lambda$ to higgsinos. Then the charged higgsino-loop induced coupling to diphotons 

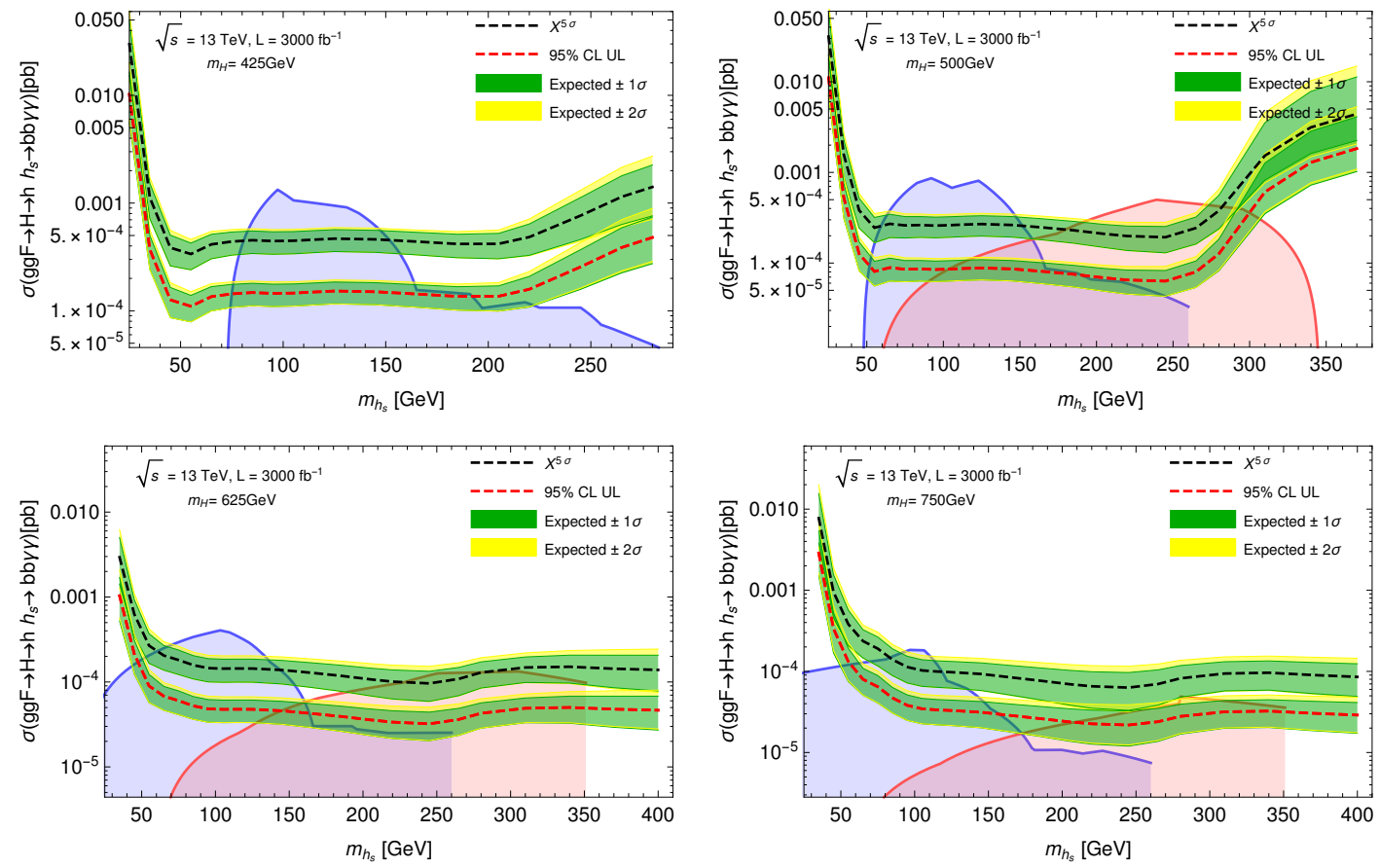

Figure 20. 95\% CL exclusion limits and $5 \sigma$ discovery cross sections for $H_{125} \rightarrow b \bar{b}$ and $H_{S} \rightarrow \gamma \gamma$ as function of $M_{H_{S}}$ for $3000 \mathrm{fb}^{-1}$ integrated luminosity and $M_{H}=425 \mathrm{GeV}$ (upper left), $M_{H}=$ $500 \mathrm{GeV}$ (upper right), $M_{H}=625 \mathrm{GeV}$ (lower left), $M_{H}=750 \mathrm{GeV}$ (lower right). The colored regions are explained in the caption of figure 8.
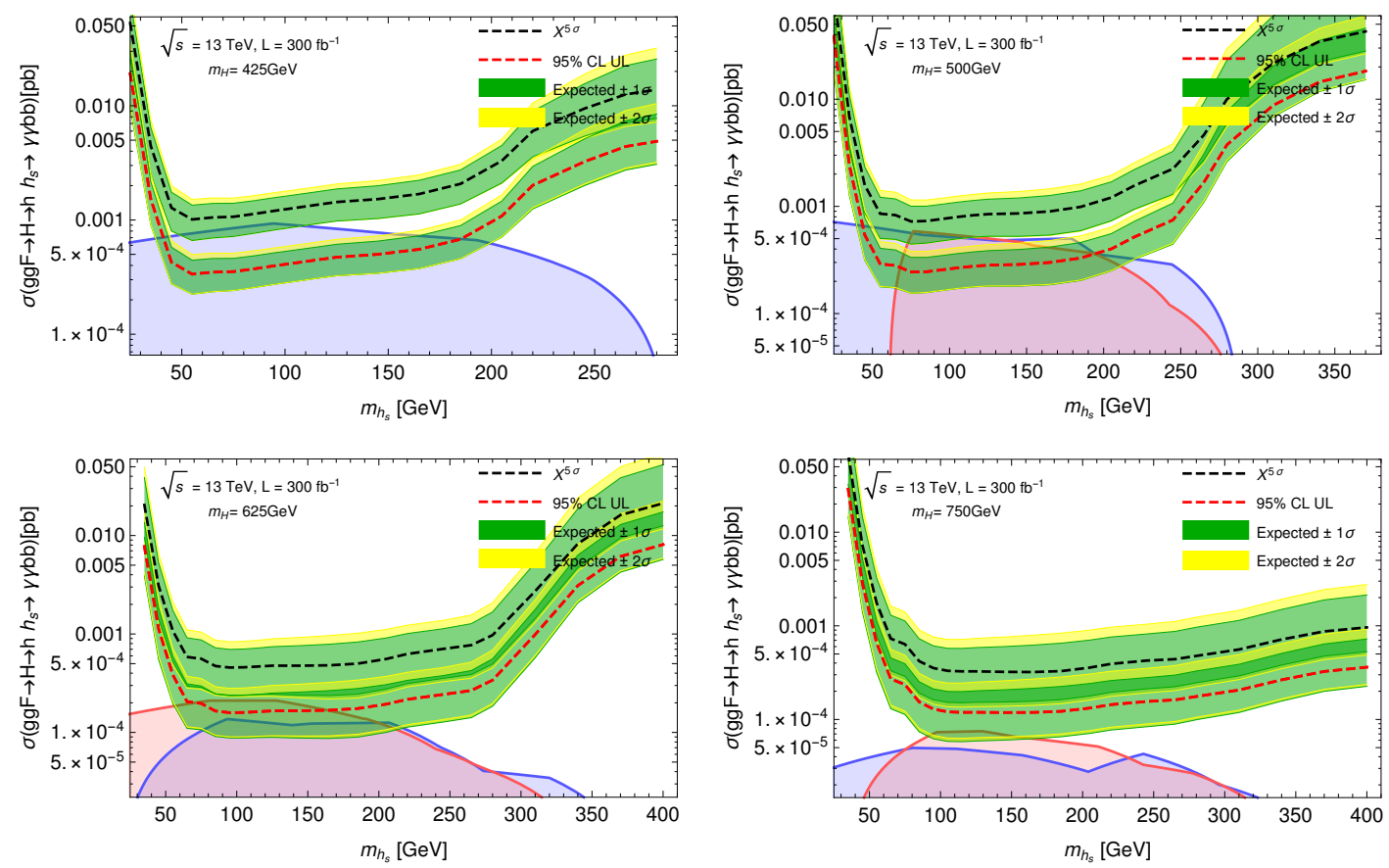

Figure 21. 95\% CL exclusion limits and $5 \sigma$ discovery cross sections for $H_{125} \rightarrow \gamma \gamma$ and $H_{S} \rightarrow b \bar{b}$ as function of $M_{H_{S}}$ for $300 \mathrm{fb}^{-1}$ integrated luminosity and $M_{H}=425 \mathrm{GeV}$ (upper left), $M_{H}=500 \mathrm{GeV}$ (upper right), $M_{H}=625 \mathrm{GeV}$ (lower left), $M_{H}=750 \mathrm{GeV}$ (lower right). The colored regions are explained in the caption of figure 8 . 

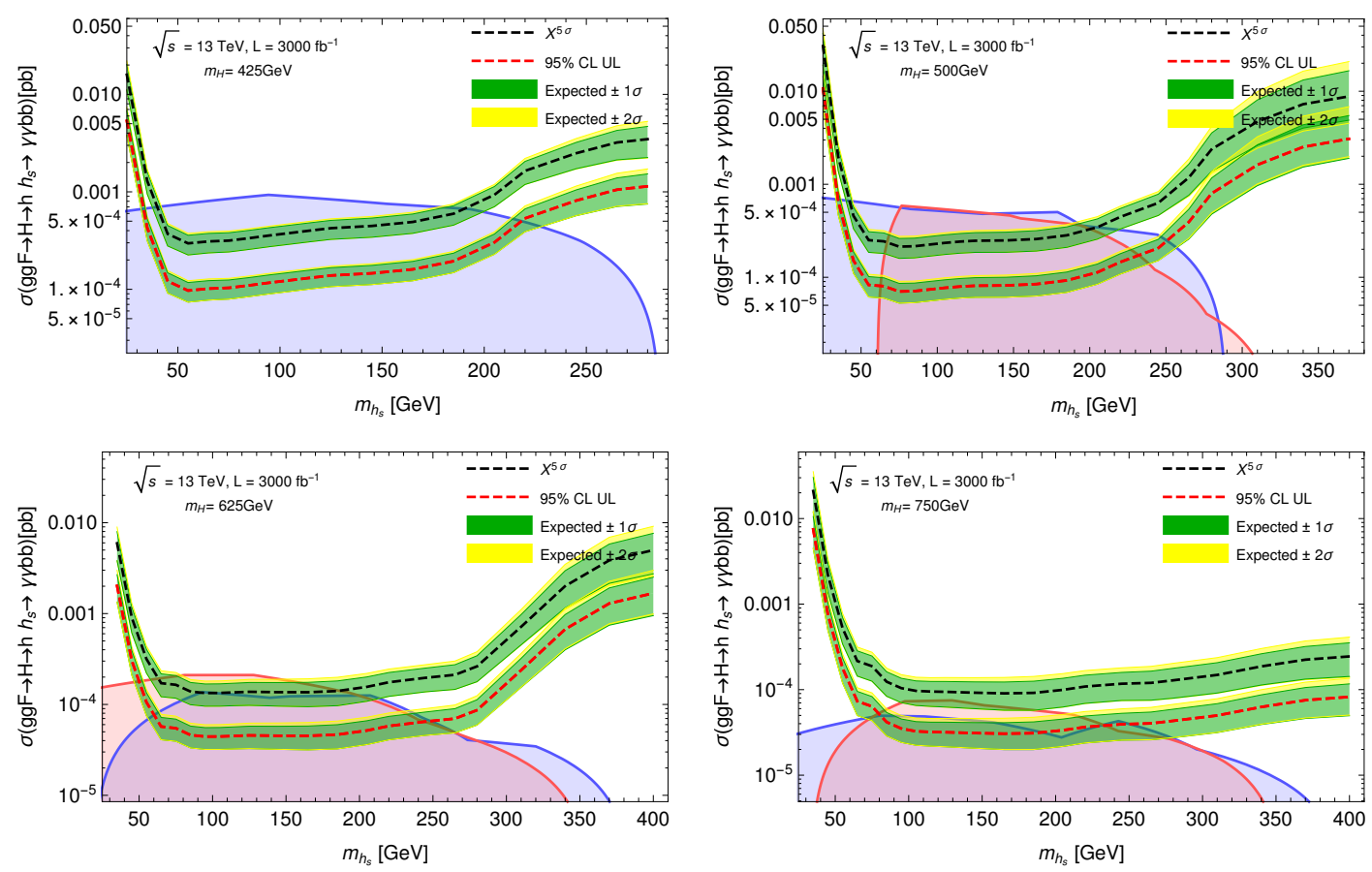

Figure 22. 95\% CL exclusion limits and $5 \sigma$ discovery cross sections for $H_{125} \rightarrow \gamma \gamma$ and $H_{S} \rightarrow b \bar{b}$ as function of $M_{H_{S}}$ for $3000 \mathrm{fb}^{-1}$ integrated luminosity and $M_{H}=425 \mathrm{GeV}$ (upper left), $M_{H}=$ $500 \mathrm{GeV}$ (upper right), $M_{H}=625 \mathrm{GeV}$ (lower left), $M_{H}=750 \mathrm{GeV}$ (lower right). The colored regions are explained in the caption of figure 8 .

can dominate, leading to a large $B R\left(A_{S} \rightarrow \gamma \gamma\right)$. The coupling $A-A_{S}-H_{125}$ is not suppressed in this case, leading to potentially large signal rates.

On the experimental side, the comparison of the upper limits on $H_{125} \rightarrow b \bar{b}$ and $H_{S} / A_{S} \rightarrow \gamma \gamma$ versus $H_{125} \rightarrow \gamma \gamma$ and $H_{S} / A_{S} \rightarrow b \bar{b}$ has a simple answer depending on $M_{H_{S} / A_{S}}$ : for $M_{H_{S} / A_{S}}<125 \mathrm{GeV}$ the search for $H_{S} / A_{S} \rightarrow \gamma \gamma$ is sensitive to smaller signal rates, whereas for $M_{H_{S} / A_{S}}>125 \mathrm{GeV}$ the search for $H_{125} \rightarrow \gamma \gamma, H_{S} / A_{S} \rightarrow b \bar{b}$ is typically sensitive to smaller signal rates. However, different regions in the parameter space of underlying models are tested by these searches.

\section{Conclusions and outlook}

Searches for resonant SM Higgs pair production are performed with considerable effort by ATLAS and CMS. As explained in the introduction searches for $g g F \rightarrow \Phi \rightarrow H_{1}+H_{2}$ can be more promising where either $H_{1}$ or $H_{2}$ can be SM-like, and the other state being possibly CP-odd (which does not affect the search methods).

This scenario is manifest in the NMSSM where the rôle of $\Phi$ is played by the MSSMlike heavy doublet, but the argument is more general. In the present paper we have studied the prospects for corresponding searches in the $b \bar{b} b \bar{b}, b \bar{b} \tau \tau$ and $b \bar{b} \gamma \gamma$ final states, including SM backgrounds. We found that significant regions in the NMSSM parameter space can be tested by these searches: 

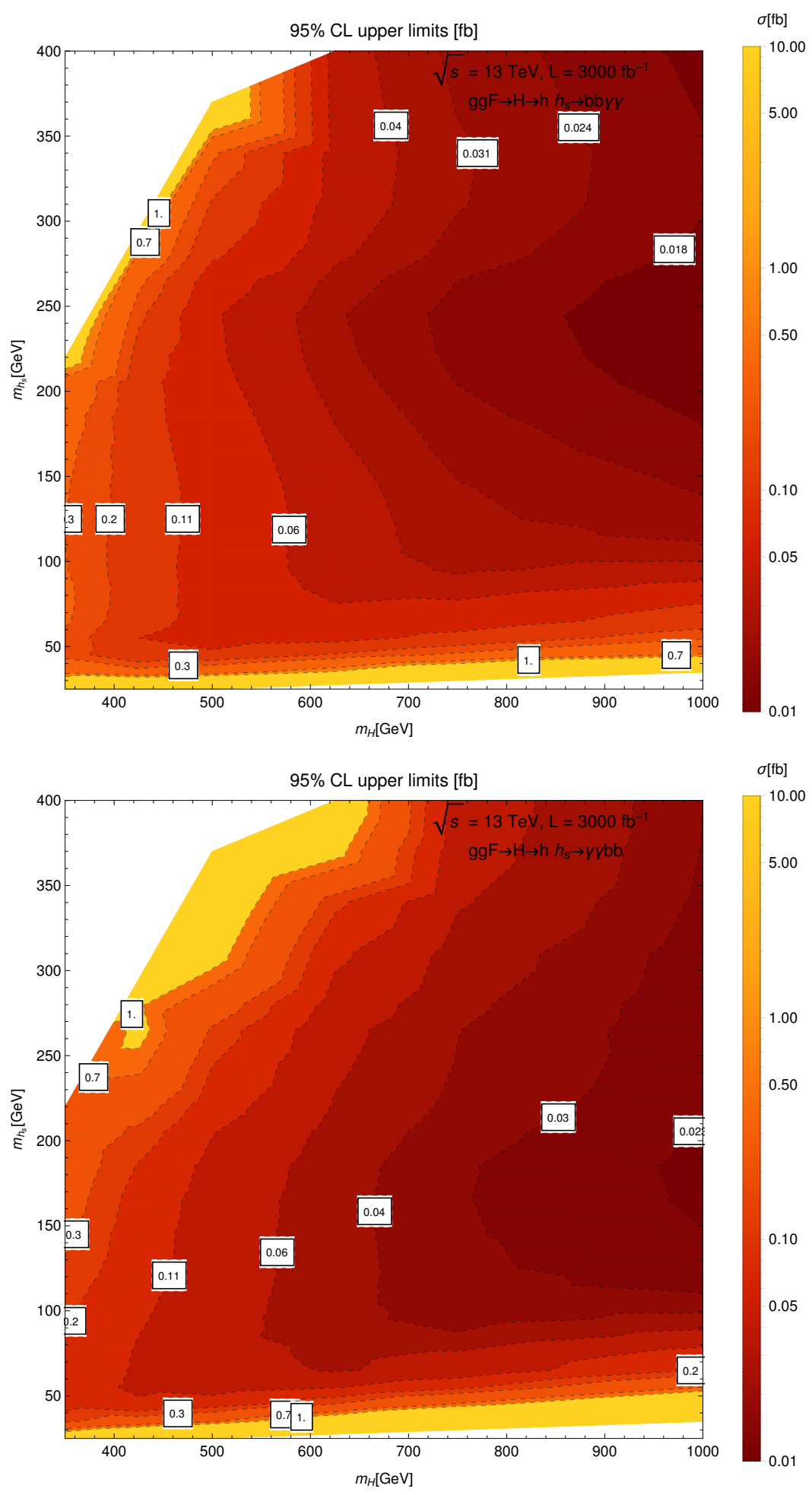

Figure 23. $95 \% \mathrm{CL}$ expected upper limits for an integrated luminosity $\mathrm{L}=3000 \mathrm{fb}^{-1}$ for the process $g g F \rightarrow H \rightarrow H_{125}(b \bar{b}) H_{S}(\gamma \gamma)$ (up) and $g g F \rightarrow H \rightarrow H_{125}(\gamma \gamma) H_{S}(b \bar{b})$ (down), using $m_{X}$ as the final discriminant. 
The NMSSM specific parameters testable by $b \bar{b} b \bar{b}$ are typically in the region $\lambda \sim$ $0.50-0.70$ (the conservative upper bound from the absence of a Landau singularity below $\left.M_{G U T}\right), \kappa \sim 0.4-0.7, \tan \beta \sim 2-3.5, \mu_{\mathrm{eff}} \sim 180 \mathrm{GeV}-290 \mathrm{GeV}, A_{\lambda} \sim 150 \mathrm{GeV}-550 \mathrm{GeV}$, $A_{\kappa} \sim-830 \mathrm{GeV}--210 \mathrm{GeV}$. An exception is the case $M_{H_{S}} \sim 85 \mathrm{GeV}-110 \mathrm{GeV}$ where LEP constraints on the coupling of $H_{S}$ to the $Z$ boson are somewhat weaker; here values of $\lambda$ down to 0.16 and $\mu$ down to $100 \mathrm{GeV}$ (together with $\tan \beta$ up to 4.5) can lead to testable points. These ranges of potentially testable parameters depend little on the total integrated luminosity, but higher luminosity increases of course the number testable parameters within these ranges. Most of the parameters testable by $b \bar{b} \gamma \gamma$ are in the same region except for $\kappa \sim 0.08-0.3, A_{\kappa} \sim-50 \mathrm{GeV}-10 \mathrm{GeV}$ which indicates that this region is non-overlapping with the one testable by $b \bar{b} b \bar{b}$.

We are convinced that the here proposed search methods can still be refined, and that the estimated sensitivities to cross sections times branching fractions presented here are conservative. This becomes clear from a comparison to the recent CMS search for resonant SM-Higgs pair production [21] in the $b \bar{b} \gamma \gamma$ final state (and actually also from a comparison to the recent CMS search [19] in the $b \bar{b} \tau \tau$ final state). Thus we hope that such promising searches will be performed in the future at the LHC.

\section{Acknowledgments}

This project has received support from the European Union's Horizon 2020 research and innovation programs ITN HiggsTools (PITN-GA-2012-316704), ITN Elusives (Marie Sklodowska-Curie grant agreement No 674896), RISE InvisiblesPlus (Marie SklodowskaCurie grant agreement No 690575), RISE NonMinimalHiggs (Marie Sklodowska-Curie grant agreement No 645722), the ERC advanced grant Higgs@LHC, and the Défi InPhyNiTi project N2P2M-SF. M.R.V. thanks Marius Wiesemann, Fady Bishara, Dirk E. Zerwas, Nikola Makovec, Sophie Henrot, Alberto Escalante and Davide Napoletano for fruitful discussions and help, and the hospitality received at the IPPP at the University of Durham, where part of this work has been done.

\section{A Functions to fit background distributions}

In this appendix we define the functions used to parametrize the total invariant mass (or $M_{X}$ ) distributions of the backgrounds to the various final states. The best choice among the functions and the values of the corresponding parameters have been obtained by maximum likelihood estimates.

\section{Four parameter Gamma distribution:}

$$
\begin{array}{ll}
f\left(M_{X} ; \alpha, \beta, \gamma, \mu\right)=\frac{\gamma}{\beta \Gamma(\alpha)} e^{-\left(\frac{\left(M_{X}-\mu\right)}{\beta}\right)^{\gamma}}\left(\frac{M_{X}-\mu}{\beta}\right)^{\alpha \gamma-1} & \text { for } M_{X} \geq \mu, \\
f\left(M_{X} ; \alpha, \beta, \gamma, \mu\right)=0 & \text { for } M_{X} \leq \mu,
\end{array}
$$




\section{Frechet distribution:}

$$
\begin{array}{ll}
f\left(M_{X} ; \alpha, \beta, \mu\right)=\frac{\alpha}{\beta} e^{-\left(\frac{\left(M_{X}-\mu\right)}{\beta}\right)^{-\alpha}}\left(\frac{M_{X}-\mu}{\beta}\right)^{-\alpha-1} & \text { for } M_{X} \geq \mu, \\
f\left(M_{X} ; \alpha, \beta, \mu\right)=0 & \text { for } M_{X} \leq \mu,
\end{array}
$$

GaussExp function: a Gaussian with an exponential tail:

$$
\begin{array}{ll}
f\left(M_{X} ; \mu, \sigma, k\right)=e^{-\frac{\left(M_{X}-\mu\right)^{2}}{2 \sigma^{2}}} & \text { for } \frac{M_{X}-\mu}{\sigma} \leq k, \\
f\left(M_{X} ; \mu, \sigma, k\right)=e^{\frac{k^{2}}{2}-\frac{k\left(M_{X}-\mu\right)}{\sigma}} & \text { for } \frac{M_{X}-\mu}{\sigma}>k .
\end{array}
$$

\section{Landau distribution:}

$$
f\left(M_{X} ; \mu, \sigma\right)=\int_{0}^{\infty} \sin (2 t) e^{-t \frac{\left(M_{X}-\mu\right)}{\sigma}-\frac{2 t}{\pi} \log (t)} d t
$$

\section{B $5 \sigma$ discovery and $95 \% \mathrm{CL}$ exclusion limits}

In this appendix we sketch the computations of $5 \sigma$ discovery and 95\% CL exclusion limits based on the $M_{X}$ distributions of the background, and the different $M_{X}$ signal distributions (depending on $M_{H_{S}}$ ), following [52].

As shown in figure 4 the $M_{X}$ distribution of the signal, after event selection and cuts, corresponds to a certain number $s_{i}$ of expected signal events per bin. (The bin size in $M_{X}$ is $20 \mathrm{GeV}$, and we have checked that the final results do not vary with this size.) From the MC simulation we know how this event number depends on the total signal cross section $\sigma_{\text {sig }}$, the integrated luminosity $\mathcal{L}_{\text {int }}$ and the acceptance $A$ times efficiency $\epsilon$ :

$$
s_{i} \sim \mathcal{L}_{\text {int }} \cdot \sigma_{s} \cdot\langle A \cdot \epsilon\rangle_{s}
$$

where $\sim$ indicates a bin-dependent proportionality factor $<1$. The backgrounds after event selection and cuts have been fitted by continuous functions of $f\left(M_{X}\right)$ normalized to 1 . Thus the number $b_{i}$ of expected background events per bin per integrated luminosity is

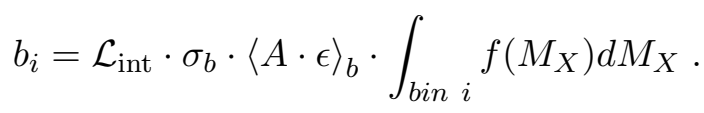

Due to the large number of simulated events the statistical uncertainties around the median values $\langle A \cdot \epsilon\rangle_{s}$ and $\langle A \cdot \epsilon\rangle_{b}$ are negligibly small, $\lesssim 1 \%$ in practically all cases.

Subsequently we use likelihood functions

$$
L\left(\sigma_{a}, \sigma_{b}\right)=\prod_{i} \frac{\left(b_{i}+\sigma_{a} s_{i}\right)^{b_{i}+\sigma_{b} s_{i}}}{\left(b_{i}+\sigma_{b} s_{i}\right) !} e^{-\left(b_{i}+\sigma_{a} s_{i}\right)}
$$

with $\left(b_{i}+\sigma_{b} s_{i}\right)$ ! interpolated by the $\Gamma$ function for non integer $\left(b_{i}+\sigma_{b} s_{i}\right)$.

For $5 \sigma$ discovery limits on $\sigma_{\text {sig }}$ we look for the value of $\sigma_{\text {sig }}$ for which the background only hypothesis is rejected at the $5 \sigma$ level. The observed number of events per bin would be 
$b_{i}+\sigma_{\text {sig }} s_{i}$, and the likelihood function corresponding to the background only hypothesis is $L\left(0, \sigma_{s i g}\right)$. As function of the number of events per bin it has its maximum at $L\left(\sigma_{\text {sig }}, \sigma_{\text {sig }}\right)$. Hence the test statistics $t_{d i s c}$ for discovery is

$$
t_{\text {disc }}=-2 \ln \frac{L\left(0, \sigma_{\text {sig }}\right)}{L\left(\sigma_{\text {sig }}, \sigma_{\text {sig }}\right)} .
$$

Following [52] the significance $Z_{\text {disc }}$ is then

$$
Z_{\text {disc }}=\sqrt{t_{\text {disc }}}
$$

and for a $5 \sigma$ discovery we determine $\sigma_{\text {sig }}$ such that $Z_{\text {disc }}=5$.

For $95 \%$ CL exclusion limits on $\sigma_{\text {sig }}$ we look for the value of $\sigma_{\text {sig }}$ for which the signal hypothesis is rejected at $95 \% \mathrm{CL}$. The observed number of events per bin would be $b_{i}$, and the likelihood function corresponding to the signal hypothesis is $L\left(\sigma_{s i g}, 0\right)$. As function of the number of events per bin it has its maximum at $L(0,0)$. Hence the test statistics $t_{\text {excl }}$ for exclusion is

$$
t_{\text {excl }}=-2 \ln \frac{L\left(\sigma_{\text {sig }}, 0\right)}{L(0,0)} .
$$

For exclusion at 95\% CL we determine $\sigma_{\text {sig }}$ such that $\sqrt{t_{\text {excl }}}=1.64$ since we consider only positive signal contributions to the number of events.

Uncertainties from the background are estimated as follows: $\pm(1-2) \sigma$ statistical uncertainties can be obtained bin by bin. To these we add linearly (to be conservative) the estimated $(1-2) \sigma$ systematic uncertainties from the overall normalisation of the background. Then the above likelihoods are recomputed with correspondingly larger and smaller values for all $b_{i}$ from which we deduce the $\pm(1-2) \sigma$ uncertainties of $\sigma_{\text {sig }}$ for the $5 \sigma$ discovery limits and $95 \%$ CL exclusion limits.

Open Access. This article is distributed under the terms of the Creative Commons Attribution License (CC-BY 4.0), which permits any use, distribution and reproduction in any medium, provided the original author(s) and source are credited.

\section{References}

[1] ATLAS collaboration, A search for resonant Higgs-pair production in the $b \bar{b} b \bar{b}$ final state in pp collisions at $\sqrt{s}=8 \mathrm{TeV}$, ATL-CONF-2014-005 (2014).

[2] ATLAS collaboration, Search for Higgs boson pair production in the $\gamma \gamma b \bar{b}$ final state using pp collision data at $\sqrt{s}=8 \mathrm{TeV}$ from the ATLAS detector, Phys. Rev. Lett. 114 (2015) 081802 [arXiv: 1406.5053] [INSPIRE].

[3] ATLAS collaboration, Search for Higgs boson pair production in the $b \bar{b} b \bar{b}$ final state from $p p$ collisions at $\sqrt{s}=8$ TeVwith the ATLAS detector, Eur. Phys. J. C 75 (2015) 412 [arXiv: 1506.00285] [INSPIRE].

[4] ATLAS collaboration, Searches for Higgs boson pair production in the $h h \rightarrow b b \tau \tau, \gamma \gamma W W^{*}, \gamma \gamma b b, b b b b$ channels with the ATLAS detector, Phys. Rev. D 92 (2015) 092004 [arXiv: 1509.04670] [INSPIRE]. 
[5] ATLAS collaboration, Search for Higgs boson pair production in the $b \bar{b} \gamma \gamma$ final state using $p p$ collision data at $\sqrt{s}=13 \mathrm{TeV}$ with the ATLAS detector, ATLAS-CONF-2016-004 (2016).

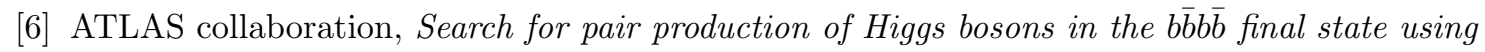
proton-proton collisions at $\sqrt{s}=13 \mathrm{TeV}$ with the ATLAS detector, ATL-CONF-2016-049 (2016).

[7] CMS collaboration, Search for two Higgs bosons in final states containing two photons and two bottom quarks in proton-proton collisions at 8 TeV, Phys. Rev. D 94 (2016) 052012 [arXiv: 1603.06896] [INSPIRE].

[8] CMS collaboration, Search for di-Higgs resonances decaying to 4 bottom quarks, CMS-PAS-HIG-14-013 (2014).

[9] CMS collaboration, Searches for heavy Higgs bosons in two-Higgs-doublet models and for $t \rightarrow$ ch decay using multilepton and diphoton final states in pp collisions at $8 \mathrm{TeV}$, Phys. Rev. D 90 (2014) 112013 [arXiv:1410.2751] [INSPIRE].

[10] CMS collaboration, Search for resonant pair production of Higgs bosons decaying to two bottom quark-antiquark pairs in proton-proton collisions at 8 TeV, Phys. Lett. B 749 (2015) 560 [arXiv: 1503.04114] [INSPIRE].

[11] CMS collaboration, Searches for a heavy scalar boson H decaying to a pair of $125 \mathrm{GeV}$ Higgs bosons hh or for a heavy pseudoscalar boson $A$ decaying to $Z h$, in the final states with $h \rightarrow \tau \tau$, Phys. Lett. B 755 (2016) 217 [arXiv:1510.01181] [INSPIRE].

[12] CMS collaboration, Search for heavy resonances decaying to two Higgs bosons in final states containing four b quarks, Eur. Phys. J. C 76 (2016) 371 [arXiv:1602.08762] [INSPIRE].

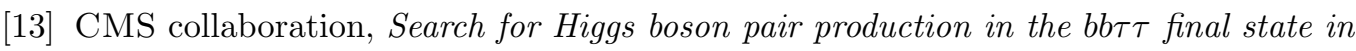
proton-proton collisions at $\sqrt{s}=8 \mathrm{TeV}$, Phys. Rev. D 96 (2017) 072004 [arXiv:1707.00350] [INSPIRE].

[14] CMS collaboration, Search for resonant pair production of Higgs bosons decaying to two bottom quark-antiquark pairs in proton-proton collisions at $13 \mathrm{TeV}$, CMS-PAS-HIG-16-002 (2016).

[15] CMS collaboration, Search for heavy resonances decaying to a pair of Higgs bosons in four $b$ quark final state in proton-proton collisions at $\sqrt{s}=13 \mathrm{TeV}$, CMS-PAS-B2G-16-008 (2016).

[16] CMS Collaboration, Search for resonant Higgs boson pair production in the $\mathrm{b} \overline{\mathrm{b}} \tau^{+} \tau^{-}$final state, CMS-PAS-HIG-16-013 (2016).

[17] CMS Collaboration, Search for resonant Higgs boson pair production in the $b \bar{b} \tau^{+} \tau^{-}$final state using 2016 data, CMS-PAS-HIG-16-029 (2016).

[18] CMS collaboration, Search for $H(b b) H(\gamma \gamma)$ decays at 13 TeV, CMS-PAS-HIG-16-032 (2016).

[19] CMS collaboration, Search for Higgs boson pair production in events with two bottom quarks and two $\tau$ leptons in proton-proton collisions at $\sqrt{s}=13 \mathrm{TeV}$, arXiv:1707.02909 [INSPIRE].

[20] CMS collaboration, Search for resonant and non-resonant Higgs boson pair production in the $b \bar{b} \ell \nu \ell \nu$ final state at $\sqrt{s}=13 \mathrm{TeV}$, CMS-PAS-HIG-17-006 (2017).

[21] CMS collaboration, Search for Higgs boson pair production in the final state containing two photons and two bottom quarks in proton-proton collisions at $\sqrt{s}=13 \mathrm{TeV}$,

CMS-PAS-HIG-17-008 (2017). 
[22] A. Djouadi, The Anatomy of electro-weak symmetry breaking. II. The Higgs bosons in the minimal supersymmetric model, Phys. Rept. 459 (2008) 1 [hep-ph/0503173] [INSPIRE].

[23] A. Djouadi and J. Quevillon, The MSSM Higgs sector at a high $M_{\mathrm{SUSY}}$ : reopening the low tan $\beta$ regime and heavy Higgs searches, JHEP 10 (2013) 028 [arXiv: 1304.1787] [INSPIRE].

[24] A. Djouadi, L. Maiani, A. Polosa, J. Quevillon and V. Riquer, Fully covering the MSSM Higgs sector at the LHC, JHEP 06 (2015) 168 [arXiv: 1502.05653] [INSPIRE].

[25] M. Maniatis, The next-to-minimal supersymmetric extension of the standard model reviewed, Int. J. Mod. Phys. A 25 (2010) 3505 [arXiv:0906.0777] [InSPIRE].

[26] U. Ellwanger, C. Hugonie and A.M. Teixeira, The next-to-minimal supersymmetric standard model, Phys. Rept. 496 (2010) 1 [arXiv:0910.1785] [INSPIRE].

[27] Z. Kang, J. Li, T. Li, D. Liu and J. Shu, Probing the CP-even Higgs sector via $\mathrm{H}_{3} \rightarrow \mathrm{H}_{2} \mathrm{H}_{1}$ in the natural next-to-minimal supersymmetric standard model, Phys. Rev. D 88 (2013) 015006 [arXiv: 1301.0453] [INSPIRE].

[28] S.F. King, M. Mühlleitner, R. Nevzorov and K. Walz, Discovery prospects for NMSSM Higgs bosons at the high-energy Large Hadron Collider, Phys. Rev. D 90 (2014) 095014 [arXiv: 1408.1120] [INSPIRE].

[29] M. Carena, H.E. Haber, I. Low, N.R. Shah and C.E.M. Wagner, Alignment limit of the NMSSM Higgs sector, Phys. Rev. D 93 (2016) 035013 [arXiv: 1510.09137] [InSPIRE].

[30] U. Ellwanger and M. Rodriguez-Vazquez, Discovery prospects of a light scalar in the NMSSM, JHEP 02 (2016) 096 [arXiv: 1512.04281] [INSPIRE].

[31] R. Costa, M. Mühlleitner, M.O.P. Sampaio and R. Santos, Singlet extensions of the standard model at LHC run 2: benchmarks and comparison with the NMSSM, JHEP 06 (2016) 034 [arXiv: 1512.05355] [INSPIRE].

[32] S. von Buddenbrock et al., Phenomenological signatures of additional scalar bosons at the LHC, Eur. Phys. J. C 76 (2016) 580 [arXiv:1606.01674] [INSPIRE].

[33] U. Ellwanger, J.F. Gunion and C. Hugonie, NMHDECAY: a Fortran code for the Higgs masses, couplings and decay widths in the NMSSM, JHEP 02 (2005) 066 [hep-ph/0406215] [INSPIRE].

[34] U. Ellwanger and C. Hugonie, NMHDECAY 2.0: an updated program for sparticle masses, Higgs masses, couplings and decay widths in the NMSSM, Comput. Phys. Commun. 175 (2006) 290 [hep-ph/0508022] [INSPIRE].

[35] G. Degrassi and P. Slavich, On the radiative corrections to the neutral Higgs boson masses in the NMSSM, Nucl. Phys. B 825 (2010) 119 [arXiv:0907.4682] [INSPIRE].

[36] http//twiki.cern.ch/twiki/bin/view/LHCPhysics/CERNYellowReportPageBSMAt13TeV.

[37] J. Alwall et al., The automated computation of tree-level and next-to-leading order differential cross sections and their matching to parton shower simulations, JHEP 07 (2014) 079 [arXiv: 1405.0301] [INSPIRE].

[38] R.V. Harlander, S. Liebler and H. Mantler, SusHi: a program for the calculation of Higgs production in gluon fusion and bottom-quark annihilation in the Standard Model and the MSSM, Comput. Phys. Commun. 184 (2013) 1605 [arXiv:1212.3249] [INSPIRE].

[39] S. Liebler, Neutral Higgs production at proton colliders in the CP-conserving NMSSM, Eur. Phys. J. C 75 (2015) 210 [arXiv:1502.07972] [INSPIRE]. 
[40] H. Mantler and M. Wiesemann, Hadronic Higgs production through NLO+PS in the SM, the 2HDM and the MSSM, Eur. Phys. J. C 75 (2015) 257 [arXiv:1504.06625] [InSPIRE].

[41] NNPDF collaboration, R.D. Ball et al., Parton distributions with QED corrections, Nucl. Phys. B 877 (2013) 290 [arXiv:1308.0598] [InSPIRE].

[42] T. Sjöstrand, S. Mrenna and P.Z. Skands, PYTHIA 6.4 physics and manual, JHEP 05 (2006) 026 [hep-ph/0603175] [INSPIRE].

[43] DELPHES 3 collaboration, J. de Favereau et al., DELPHES 3, A modular framework for fast simulation of a generic collider experiment, JHEP 02 (2014) 057 [arXiv:1307.6346] [INSPIRE].

[44] M. Cacciari and G.P. Salam, Dispelling the $N^{3}$ myth for the $k_{t}$ jet-finder, Phys. Lett. B 641 (2006) 57 [hep-ph/0512210] [INSPIRE].

[45] ATLAS collaboration, Expected performance of the ATLAS b-tagging algorithms in Run-2, ATL-PHYS-PUB-2015-022 (2015).

[46] M. Cacciari, M. Czakon, M. Mangano, A. Mitov and P. Nason, Top-pair production at hadron colliders with next-to-next-to-leading logarithmic soft-gluon resummation, Phys. Lett. B 710 (2012) 612 [arXiv:1111.5869] [INSPIRE].

[47] M. Czakon and A. Mitov, Top++: a program for the calculation of the top-pair cross-section at hadron colliders, Comput. Phys. Commun. 185 (2014) 2930 [arXiv:1112.5675] [InSPIRE].

[48] P. Bärnreuther, M. Czakon and A. Mitov, Percent level precision physics at the tevatron: first genuine NNLO QCD corrections to $q \bar{q} \rightarrow t \bar{t}+X$, Phys. Rev. Lett. 109 (2012) 132001 [arXiv: 1204.5201] [INSPIRE].

[49] M. Czakon and A. Mitov, NNLO corrections to top-pair production at hadron colliders: the all-fermionic scattering channels, JHEP 12 (2012) 054 [arXiv:1207.0236] [INSPIRE].

[50] M. Czakon and A. Mitov, NNLO corrections to top pair production at hadron colliders: the quark-gluon reaction, JHEP 01 (2013) 080 [arXiv: 1210.6832] [INSPIRE].

[51] M. Czakon, P. Fiedler and A. Mitov, Total top-quark pair-production cross section at hadron colliders through $O\left(\alpha_{S}^{4}\right)$, Phys. Rev. Lett. 110 (2013) 252004 [arXiv: 1303.6254] [InSPIRE].

[52] G. Cowan, K. Cranmer, E. Gross and O. Vitells, Asymptotic formulae for likelihood-based tests of new physics, Eur. Phys. J. C 71 (2011) 1554 [Erratum ibid. C 73 (2013) 2501] [arXiv: 1007.1727] [INSPIRE]. 